ARTICLE

https://doi.org/10.1038/s41467-019-13832-9

\title{
Sestrin prevents atrophy of disused and aging muscles by integrating anabolic and catabolic signals
}

Jessica Segalés ${ }^{1,2}$, Eusebio Perdiguero (10) 1,12, Antonio L. Serrano 1,12, Pedro Sousa-Victor 1,10,12, Laura Ortet ${ }^{1}$, Mercè Jardí , Andrei V. Budanov ${ }^{3,4}$, Laura Garcia-Prat ${ }^{1,2,11}$, Marco Sandri ${ }^{5}$, David M. Thomson ${ }^{6}$, Michael Karin Jun Hee Lee $\mathbb{C i}^{8}$ \& Pura Muñoz-Cánoves ${ }^{1,2,9 *}$

A unique property of skeletal muscle is its ability to adapt its mass to changes in activity. Inactivity, as in disuse or aging, causes atrophy, the loss of muscle mass and strength, leading to physical incapacity and poor quality of life. Here, through a combination of transcriptomics and transgenesis, we identify sestrins, a family of stress-inducible metabolic regulators, as protective factors against muscle wasting. Sestrin expression decreases during inactivity and its genetic deficiency exacerbates muscle wasting; conversely, sestrin overexpression suffices to prevent atrophy. This protection occurs through mTORC1 inhibition, which upregulates autophagy, and AKT activation, which in turn inhibits FoxO-regulated ubiquitin-proteasomemediated proteolysis. This study reveals sestrin as a central integrator of anabolic and degradative pathways preventing muscle wasting. Since sestrin also protected muscles against aging-induced atrophy, our findings have implications for sarcopenia.

\footnotetext{
${ }^{1}$ Department of Experimental \& Health Sciences, University Pompeu Fabra, CIBERNED, 08003 Barcelona, Spain. ${ }^{2}$ Centro Nacional de Investigaciones Cardiovasculares, 28019 Madrid, Spain. ${ }^{3}$ School of Biochemistry and Immunology, Trinity Biomedical Sciences Institute, Trinity College Dublin, Dublin D02 R590, Ireland. ${ }^{4}$ Engelhardt Institute of Molecular Biology, Center for Precision Genome Editing and Genetic Technologies for Biomedicine, 119991 Moscow, Russia. ${ }^{5}$ Department of Biomedical Science, University of Padova, 35100 Padova, Italy. ${ }^{6}$ Department of Physiology and Developmental Biology, Brigham Young University, Provo, UT 84602, USA. ${ }^{7}$ Department of Pharmacology, University of California San Diego, La Jolla, CA 92093, USA. ${ }^{8}$ Department of Molecular and Integrative Physiology, University of Michigan, Ann Arbor, MI 48109-2200, USA. ${ }^{9}$ ICREA, 08003 Barcelona, Spain. ${ }^{10}$ Present address: Instituto de Medicina Molecular (iMM), Faculdade de Medicina, Universidade de Lisboa, 1649 Lisbon, Portugal. ${ }^{11}$ Present address: Princess Margaret Cancer Centre, University Health Network, Toronto M5G $1 \mathrm{~L} 7$ ON, Canada. ${ }^{12}$ These authors contributed equally: Eusebio Perdiguero, Antonio L. Serrano, Pedro Sousa-Victor. ${ }^{\star}$ email: pura.munoz@upf.edu
} 
T he control of mass in adult skeletal muscle is determined by a dynamic balance between anabolic and catabolic processes triggered by changes in activity or pathological conditions ${ }^{1}$. Muscle hypertrophy is associated with increased protein synthesis induced by activated AKT and mammalian target of rapamycin complex 1 (mTORC1) pathways ${ }^{1}$. Unlike muscle hypertrophy, muscle atrophy always involves a proteostatic shift in favor of catabolic versus anabolic processes ${ }^{1,2}$. Chief among these implicated catabolic changes is the activation of the autophagy-lysosome and ubiquitin-proteasome pathways, in particular the induction of muscle-specific ubiquitin ligases of the atrophy-related gene family, also known as atrogenes. Atrogenes, which are regulated by FoxO transcription factors (TF), remove proteins and organelles in atrophying fibers, whereas anabolic myofiber growth depends on AKT and mTOR activation ${ }^{3-7}$. AKT phosphorylates FoxO TF and impedes their nuclear activity, thus suppressing FoxO-dependent atrogene expression ${ }^{8}$. A complex scenario is thus emerging in which catabolic signaling connects with biosynthetic pathways during muscle atrophy, although little is known about how these connections are established.

Skeletal muscle atrophy is a major health problem and is the consequence of a wide variety of pathological conditions, including inactivity (immobilization or nerve injury), chronic diseases, and neuromuscular disorders. Muscle atrophy also complicates many aging-associated diseases, lowers life quality, and increases mortality. Regardless of the driving origin, muscle atrophy always involves loss of muscle mass, strength, and function ${ }^{1,2}$. Preventing or reversing muscle atrophy is therefore of the utmost importance, yet the mechanisms driving muscle atrophy are largely unknown.

Through a transcriptomic/bioinformatic screen for potential atrophy regulators, we identify the sestrin genes, particularly sestrin1 (but also sestrin 2), as genes downregulated rapidly in several models of muscle atrophy in vivo, including disuse, denervation, and aging (sarcopenia). Sestrins are a family of stress-inducible metabolic regulators that are conserved throughout metazoans ${ }^{9}$. Cell-based studies showed that sestrins have an antioxidant function that suppresses reactive oxygen species (ROS) $)^{9,10}$. Genetic studies of Drosophila sestrin (dSesn) revealed that, by activating AMPK, dSesn also functions as negative regulator of dTORC1, leading to several age-related pathologies ${ }^{11}$. Similar age-associated metabolic defects are also observed in cSesn-mutated Caenorhabditis elegans ${ }^{12}$. Recent studies indicate that mouse sestrins attenuate obesity-associated metabolic liver diseases, such as insulin resistance and steatohepatitis by suppressing oxidative stress or modulating AMPK/ mTORC1 activity $10,13-15$. However, the role of mammalian sestrins in the regulation of muscle mass control is unknown, despite skeletal muscle being the site of maximal sestrin 1 (Sesn1) expression in humans and mice ${ }^{16}$.

In this study, we have made the surprising observation that not all catabolic activities are enhanced during muscle atrophy; rather, while proteasome activity is induced in response to inactivity, autophagy is blunted. Using sestrin gain-of-function and ablation approaches in mice, we find that sestrin preserves muscle mass and force in atrophying conditions by coordinating anabolic and catabolic pathways. This protective effect extends to age-induced muscle atrophy. Our results reveal that sestrin is a key instructor of skeletal muscle mass.

\section{Results}

Sestrins protect from disuse-induced muscle atrophy. Through a bioinformatic analysis of the atrophy-associated transcriptome, we searched for potential growth and atrophy regulators. Sesn 1 was identified as one of six genes dysregulated in distinct models of muscle wasting in vivo, such as disuse and denervation (Fig. 1a and Supplementary Data 1). Sesn1 belongs to a conserved stressinducible family of proteins with antioxidant and metabolic functions, which in vertebrates are encoded by the Sesn1, Sesn2, and Sesn3 loci ${ }^{9}$. Sesn1, among sestrins, is highly expressed in mammalian skeletal muscle ${ }^{16}$ (Supplementary Fig. 1a). Considering that sestrins control mTOR and AKT pathways $9,11,14,17,18$, critical regulators of muscle function ${ }^{1,2}$, we examined the influence of Sesn1 downregulation on muscle atrophy.

Sesn1 RNA and protein expression decreased in mouse skeletal muscle after immobilization-induced limb inactivity (Fig. 1b), correlating with muscle atrophy and loss of force (Fig. 1c-f, white and gray charts). To investigate the involvement of Sesn1 in disuse atrophy, we generated transgenic mice overexpressing human Sesn 1 (96\% homologous to murine Sesn1) in skeletal muscle

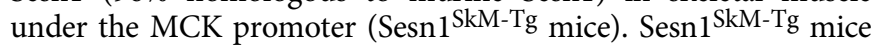
expressed Sesn1 in both fast and slow muscles, without changes in Sesn2 and Sesn3 expression (Supplementary Fig. 1b, c), were of normal weight, and showed no obvious muscle abnormalities under normal conditions when compared to littermate Sesn 1 WT (wild-type, WT) mice (Supplementary Fig. 1d, e). However,

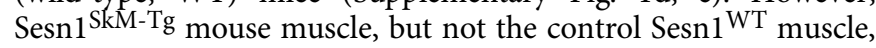
was strongly protected against all measures of disuse atrophy, including muscle weight, myofiber size, fibrosis index, and force production by the extensor digitorum longus (EDL) (Fig. 1c, d, navy and blue charts, and Supplementary Fig. 1f) and soleus muscles (Supplementary Fig. 1g, navy and blue charts). No changes in myonuclear number were observed (Supplementary Fig. 1h). Importantly, Sesn 1 also protected from atrophy induced by denervation, as shown by the preservation of myofiber size and muscle force in Sesn 1 SkM-Tg mice compared to Sesn 1 WT mice upon sciatic denervation (Supplementary Fig. 1i, j).

Given the high sequence homology of mammalian sestrins (Sesn1-3), and to discriminate whether the atrophy-preventing effect was specific for Sesn1 or could also be exerted by another sestrin family member, we tested muscle atrophy protection with a transgenic mouse line overexpressing human Sesn2 (92\% homologous to murine Sesn2) in skeletal muscle (Sesn2 $2 \mathrm{kM}-\mathrm{Tg}$ mice), in comparison to littermate control Sesn2WT mice

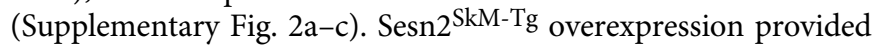
similar protection from disuse atrophy and force loss (Fig. 1e, f and Supplementary Fig. 2d), indicating that, upon overexpression, Sesn 1 and Sesn 2 are equally effective at preventing immobilization-induced muscle atrophy.

Transgenic Sesn $1 / 2$ expression throughout development has the potential to produce adaptive effects unrelated to sestrin activity that may mask sestrin functions in the adult. To exclude this, we overexpressed Sesn1 and Sesn2 in skeletal muscle of 4month-old WT mice via adeno-associated virus (AAV) transduction (Supplementary Fig. 2e, f). Consistent with the results from transgenic mice, AAV-based Sesn1 or Sesn2 overexpression in WT muscle also prevented disuse-induced muscle atrophy and weakness (Fig. 1g, h). Sestrins may function as antioxidants through their oxidoreductase activity ${ }^{10,19}$, prompting us to test the effect of an oxidoreductase-disrupting C130 mutation in Sesn ${ }^{10,19}$. Overexpression of Sesn1-C130S still impeded disuse muscle atrophy (Supplementary Fig. 2g), indicating that the protective action of sestrin is independent of its antioxidant function.

Loss of Sesn 1 aggravates disuse-induced muscle atrophy. Of the three sestrins, Sesn1 is the main form expressed in muscle $^{10,16,19}$ (Supplementary Fig. 1a). We therefore analyzed mice deficient in Sesn1 (Sesn1 ${ }^{\mathrm{KO}}$ mice) ${ }^{20}$ in comparison to their WT controls. Although these mice showed no detectable 
a

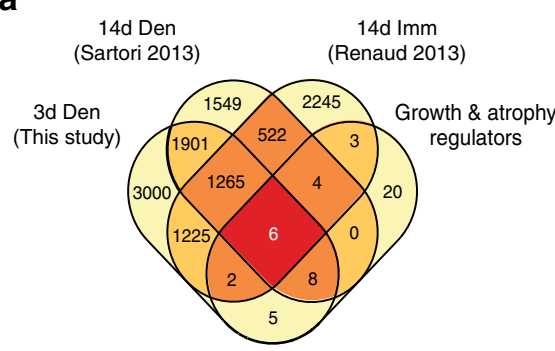

C
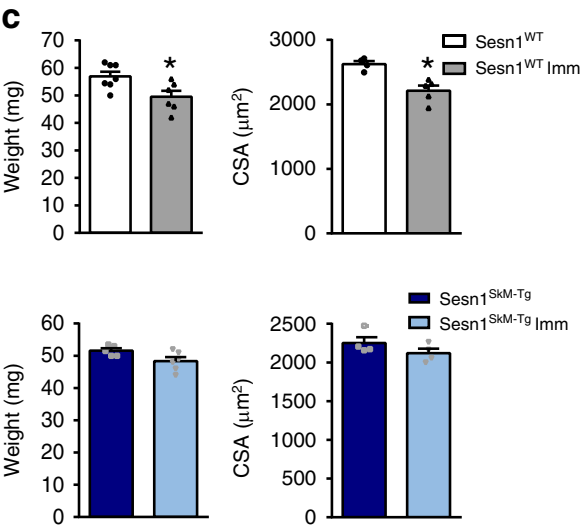

e
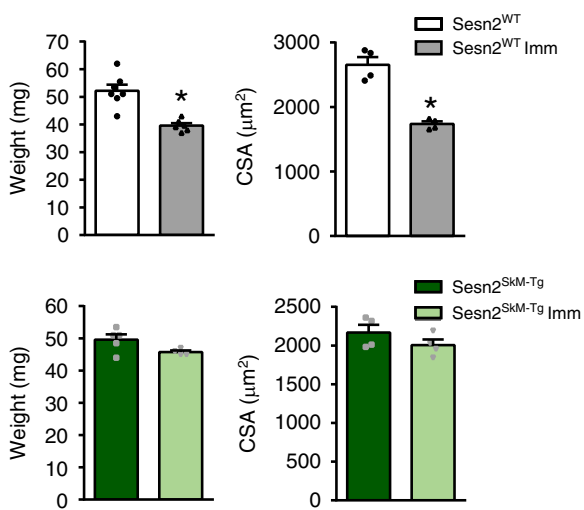

g

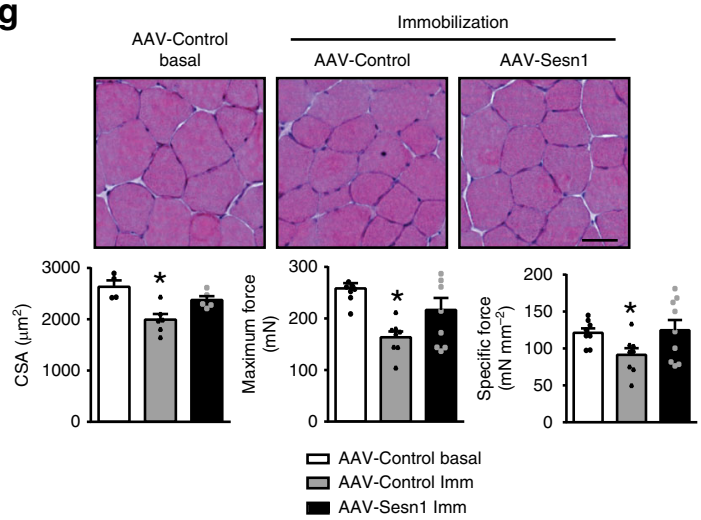

alterations in muscle weight, myofiber size, or force under basal conditions (Supplementary Fig. 3a-d), they showed more pronounced myofiber atrophy and force loss in EDL and soleus muscles in response to inactivity (Fig. 2a-d). No changes in Sesn $2 /$ Sesn 3 levels were found in muscles of Sesn $1 \mathrm{KO}^{\mathrm{m}}$ mice (Supplementary Fig. 3e); Sesn1 is thus critical for preventing muscle wasting.

f b
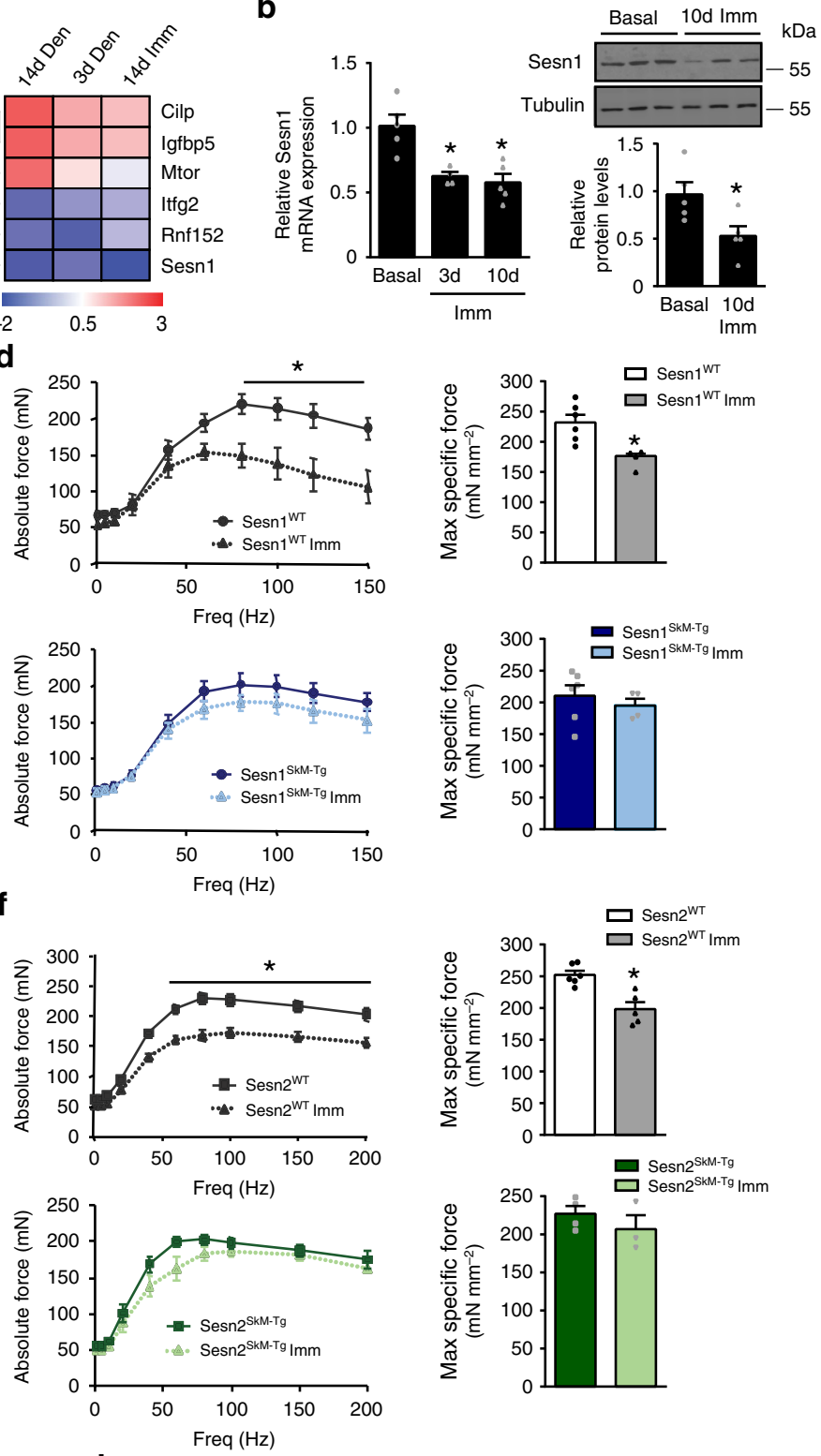

h
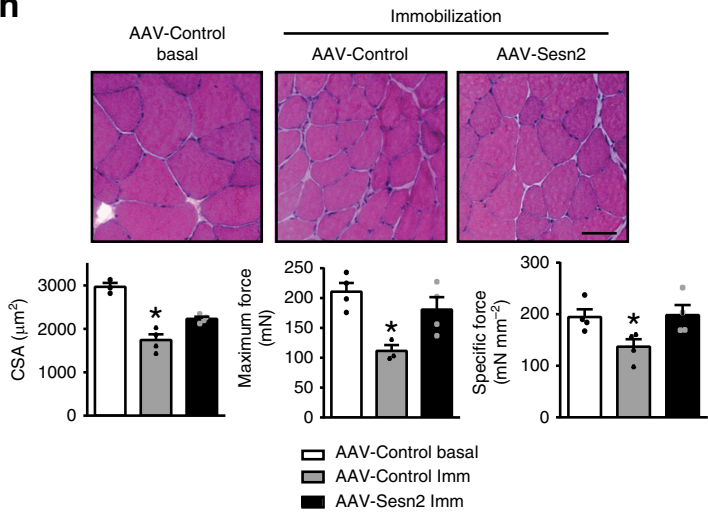

We next evaluated whether sestrin provided equal protection to distinct types of muscle fibers. Disuse muscle atrophy was associated with muscle fiber-type switching from IIA/IIX to IIB fibers in WT mice and in Sesn $1^{\mathrm{SkM}-\mathrm{Tg}}$ mice (Supplementary Fig. 3f). Preservation of muscle mass upon Sesn1 overexpression and increased atrophy after Sesn 1 deletion was observed in type IIA/IIX and type IIB fibers (Supplementary Fig. 3f, g), indicating 
Fig. 1 Sestrins prevent disuse-induced skeletal muscle atrophy. a Left Venn diagram showing overlap between a gene set of growth and atrophy regulators (see the "Methods" section) and dysregulated genes in immobilized (Imm) or denervated (Den) muscles reported in published muscle atrophy models or identified in our RNAseq comparison. Right Heat map for the six genes dysregulated in all gene sets analyzed. b Analysis of Sesn1 mRNA (left) and protein (right) in tibialis anterior (TA) muscles from non-immobilized (basal) and immobilized (Imm) limbs of WT mice for the indicated number of

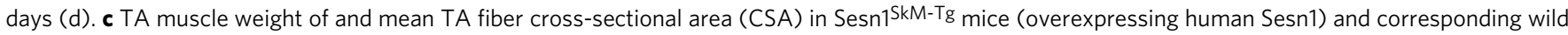
type (WT) mice (Sesn1 ${ }^{\mathrm{WT}}$ ) in basal conditions and after 10 days of limb immobilization. $\mathbf{d}$ Force measurements in extensor digitorum longus (EDL) muscle of Sesn1WT and Sesn1 ${ }^{\mathrm{SkM} \text {-Tg }}$ mice in basal conditions and after 10 days of limb immobilization. Charts show force-frequency curves (left) and maximum

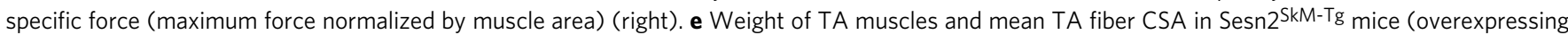
human Sesn2) and corresponding WT mice (Sesn2WT) in basal conditions and after 10 days of limb immobilization. f Force measurements in EDL muscles

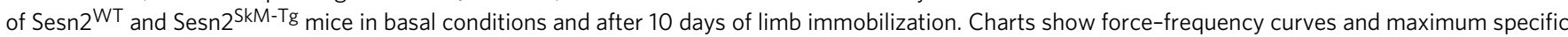
force. $\mathbf{g}$ Histology and muscle force in EDL muscles of young mice transduced with AAV-Sesn1 or AAV-Control followed 4 days later by limb immobilization for 10 additional days. The upper panels show representative images of hematoxylin/eosin (H/E) staining in basal and immobilized muscles. Scale bar $=50 \mu \mathrm{m}$. The charts show fiber size (CSA), maximum force, and specific force. $\mathbf{h}$ Histology and muscle force in EDL muscles transduced with AAV-Sesn2 or AAV-Control and treated as described in $\mathbf{g}$. Scale bar $=50 \mu \mathrm{m}$. All data are shown as mean with SEM. Statistical comparisons by unpaired two-tailed Student's $t$-test ( ${ }^{*} p<0.05$ vs. basal conditions). Sample numbers were $n=4-6$ mice per group for $\mathbf{c}-\mathbf{f}$ and $n=4$ mice per condition for g, h. Source data are provided as a Source Data file.

that sestrin protects against muscle atrophy independently of fiber-type changes.

Sestrin blunts FoxO-dependent atrogenes in disused muscle. To interrogate the mechanistic basis of sestrin-mediated protection against muscle atrophy, we performed RNAseq analysis on control and immobilized TA muscles of all available mouse

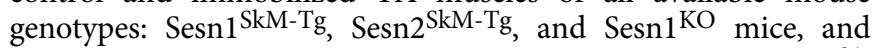
their respective WT counterparts. Like other atrophy models ${ }^{21}$, muscle immobilization enriched gene expression signatures for apoptosis, inflammation, cell-cycle inhibition, and anabolic regulation in all mouse groups (Fig. 3a and Supplementary Data 2). Interestingly, the association between immobilization and anabolic signaling hallmarks (PI3K/AKT/mTORC1 regulation) was strengthened by Sesn $1 / 2$ overexpression, whereas these hallmarks correlated negatively with Sesn1 deficiency (Fig. 3a).

We also performed a hierarchical clustering analysis to identify genes regulated by both immobilization and sestrins (Supplementary Fig. 4a). We focused on gene clusters whose immobilizationdependent induction was increased or decreased by Sesn overexpression (Clusters A and B) but were behaving contrarily in Sesn1-deficient muscles (Clusters C and D) (Supplementary Fig. 4a and Supplementary Data 3). Intersection between clusters $\mathrm{A} / \mathrm{C}$ and clusters B/D yielded a gene set (defined as Sestrin-regulated genes) that was highly enriched in canonical pathways implicated in ubiquitin-proteasome-mediated proteolysis, including proteasome subunits and the E3 ubiquitin-ligase atrogenes MuRF1 (Trim63) and Atrogin1 (Fbxo32) ${ }^{6,22-24}$ (Supplementary Fig. 4a and Supplementary Data 3). Other proteostasis pathways, such as macroautophagy-lysosome degradation, were also enriched (Supplementary Fig. 4b). Interestingly, genes regulated by immobilization and sestrins were often found to contain binding sites for FoxO, Myc/Maz, and Sp1 TF (Fig. 3b). FoxO TF-binding sites were highly enriched in atrogenes and autophagy genes present in the sestrin-regulated gene set (Supplementary Fig. $4 \mathrm{c}$, left and right). Of note, a significant overlap was found between sestrin-regulated atrogenes and a previously described list of FoxO-regulated atrogenes ${ }^{24}$ (Supplementary Fig. 4c, middle). These results are consistent with the former findings that upregulation of atrogenes, particularly Atrogin 1 and MuRF1, is directed by FoxO3a and required for muscle atrophy $3,6,7,24$.

Based on these findings, we examined the status of AKT-FoxO signaling in immobilized muscle of Sesn $1 / 2$ transgenic mice. Immobilized muscles of Sesn $1^{\text {SkM-Tg }}$ and Sesn $2^{\text {SkM-Tg mice had }}$ higher levels of AKT and FoxO3 phosphorylation than WT counterparts (Fig. 3c, d; Supplementary Fig. 5a). Notably, sestrin overexpression in muscle upregulated both PDK1-dependent AKT phosphorylation at Thr308 and mTORC2-dependent phosphorylation at Ser473 (Fig. 3d). Phosphorylation at both sites activates AKT kinase activity, while AKT-mediated phosphorylation of FoxO inhibits its transcriptional activity by retaining it in the cytoplasm $^{8}$. Consequently, immobilized Sesn $1 / 2^{\text {SkM-Tg muscle }}$ exhibited lower expression of well-known FoxO targets ${ }^{24}$ such as Atrogin1 and MuRF1 (Fig. 3e and Supplementary Fig. 5b), consistent with reduced FoxO transcriptional activity and with the transcriptome studies (Supplementary Figs. 4b, c and 5c). In contrast, disused muscle of Sesn $1{ }^{\mathrm{KO}}$ mice expressed higher levels of these atrogenes (Supplementary Fig. 5d). In line with atrogenemediated upregulation of ubiquitin-proteasome activity, the immobilization-induced increase in chymotrypsin-like proteasome activity in muscles of WT mice was blunted by sestrin overexpression (Fig. 3f). Conversely, blocking proteasome activity with bortezomib prevented the disuse atrophy in WT mice (Supplementary Fig. 5e). These results suggest that sestrins protect disused muscles from wasting, at least in part, by repressing the induction of FoxO-regulated atrogenes encoding muscle proteolytic enzymes.

Involvement of FoxO in sestrin-mediated muscle protection was confirmed through genetic modulation of FoxO. Musclespecific overexpression of constitutively active FoxO3 (FoxO3 $\mathrm{TM}^{7}$ ) abolished the protective effect of Sesn1 against muscle

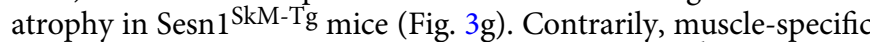
genetic deletion of all three FoxO genes (FoxO1,3,4 SkM-KO tripleknockout mice) (Supplementary Fig. 5f) produced strong protection against inactivity-induced muscle atrophy-like Sesn1/ 2 overexpression (Fig. 3h, see Fig. 1 for comparison). AKT activation by sestrin thus blocks ubiquitin-proteasome-dependent proteolysis via FoxO-signaling inhibition, subsequently attenuating muscle wasting during disuse.

Sestrin coaxes autophagy by blunting mTORC1 to protect muscle. Gene set enrichment analysis (GSEA) also identified mTORC1-signaling regulation as another hallmark of sestrin modulation in muscle (Fig. 4a). mTORC1 is a major anabolic factor, classically associated with promotion of muscle growth and hypertrophy. Although mTORC1 was originally thought to decline during muscle atrophy ${ }^{1,2}$, we found sustained-and even increased-mTORC1 activity in immobilized atrophic muscles of non-transgenic mice, as revealed by phosphorylation of the mTORC1 downstream targets S6 and ULK (Fig. 4b). Overexpression of Sesn1 or Sesn2, which restores muscle mass in the disuse condition, strongly inhibited mTORC1 signaling 
a

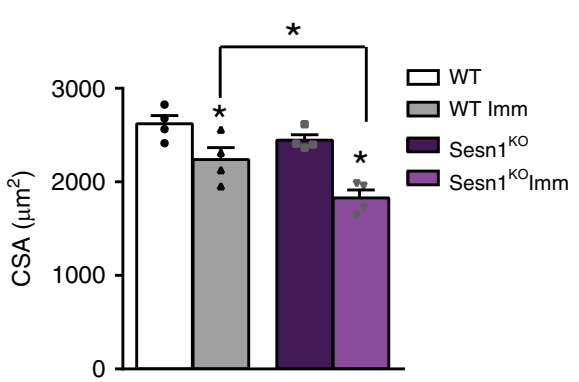

C

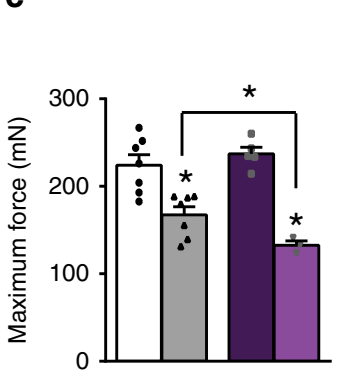

EDL

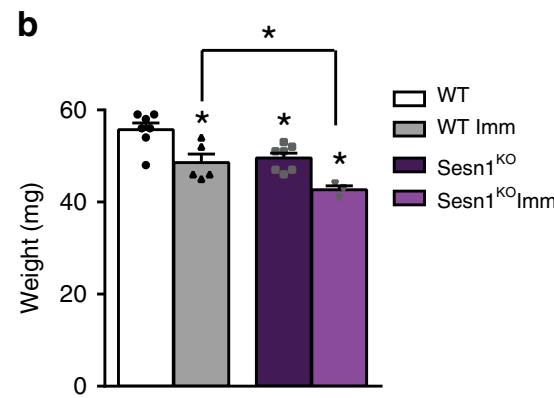

d $\quad$ Soleus
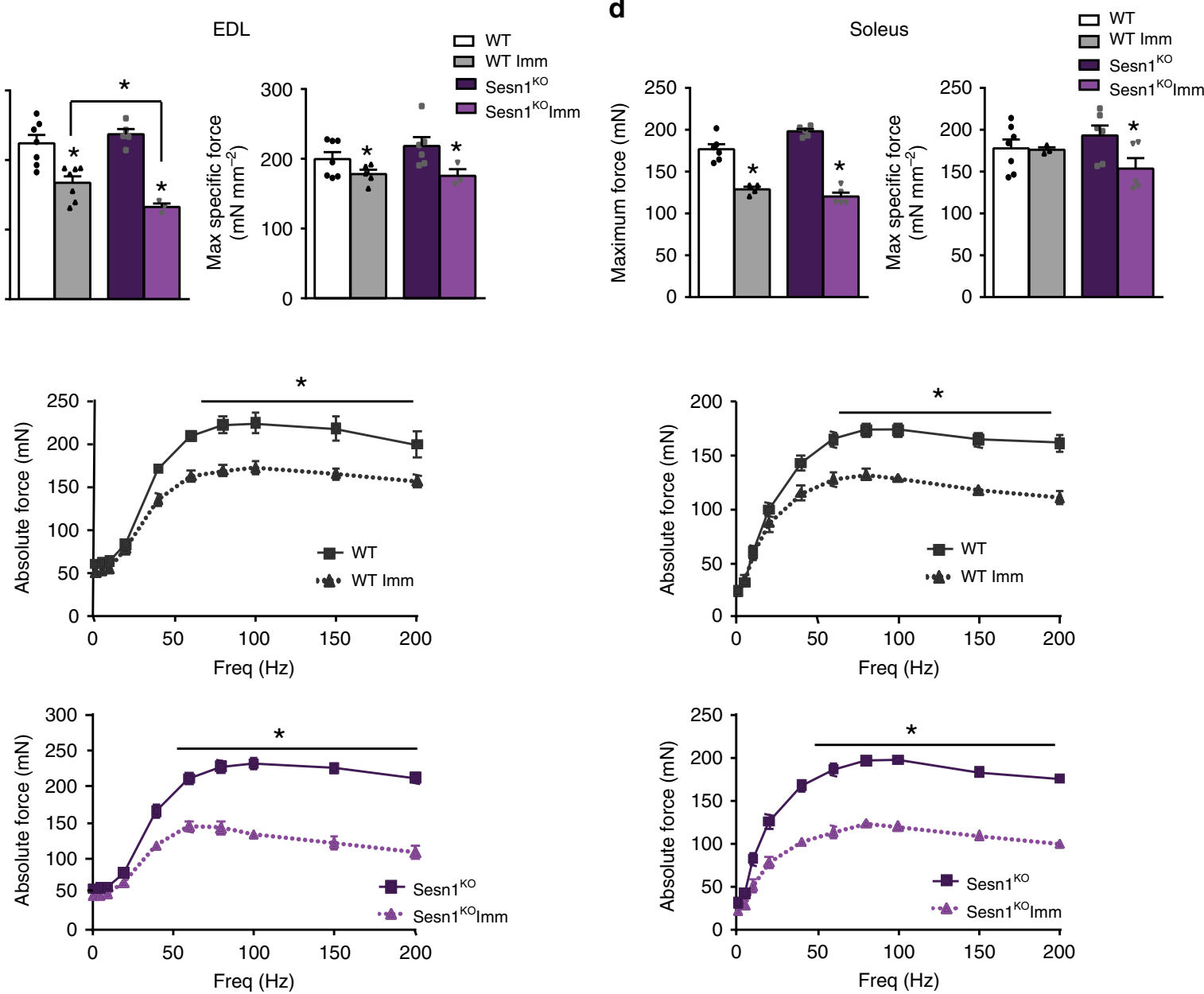

Fig. 2 Loss of sestrin1 exacerbates disuse-induced muscle atrophy. a Mean CSA of TA fibers from WT and Sesn1 ${ }^{\mathrm{KO}}$ mice in basal conditions and after 10 days of limb immobilization. b TA muscle weight in WT and Sesn ${ }^{\mathrm{KO}}$ mice in basal conditions and after 10 days of limb immobilization. $\mathbf{c}$, $\mathbf{d}$ Force measurements in EDL $\mathbf{c}$ and soleus $\mathbf{d}$ muscles of WT and Sesn1 ${ }^{\mathrm{KO}}$ mice in basal conditions and after 10 days of limb immobilization. Charts show maximum and specific force (top) and force-frequency curves (bottom). All data are shown as mean with SEM. Comparisons by unpaired two-tailed Student's $t$-test $\left({ }^{\star} p<0.05\right) . N=3-7$ mice per genotype and condition. Source data are provided as a Source Data file.

(i.e. reduced S6 and ULK1 phosphorylation) after immobilization (Fig. 4b), whereas Sesn1 ${ }^{\mathrm{KO}}$ muscle showed constitutive mTORC1-signaling activation even in resting muscle (Supplementary Fig. 6a). Therefore, muscle immobilization unexpectedly conveys mTORC1 activation, whereas sestrins strongly downregulate it.

mTORC1-mediated ULK1 phosphorylation inhibits autophagy ${ }^{13,25}$, another major protein and organelle degradation pathway. The autophagy pathway was also found in the sestrinregulated gene set, which included many autophagy-related genes enriched in FoxO TF-binding sites (Supplementary Fig. 4b, c and
Supplementary Data 3). Skeletal muscle autophagy flux was determined in vivo using colchicine, which blocks autophagosome degradation ${ }^{26}$. Colchicine-triggered marked accumulation of the autophagosome marker LC3-II in WT and sestrin-overexpressing muscles (Fig. 4c, left panel, and Supplementary Fig. 6b), indicating that basal autophagy flux is active in all these tissues, being barely affected by Sesn $1 / 2$ overexpression in non-disuse conditions. However, muscle immobilization in WT mice strongly decreased colchicine-dependent LC3-II accumulation (Fig. 4c, right panel, and Supplementary Fig. 6c), indicating that autophagy flux was attenuated. Importantly, autophagy flux was preserved in 
a

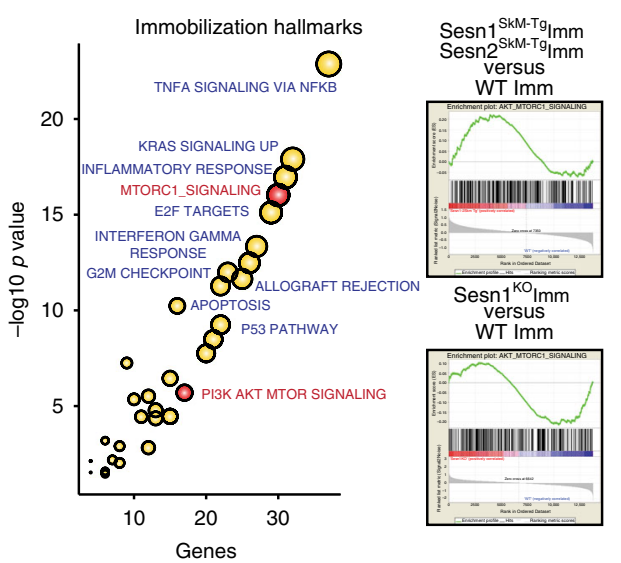

C

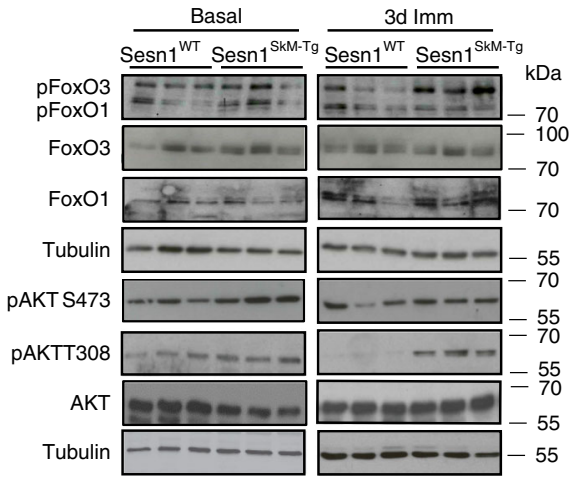

d

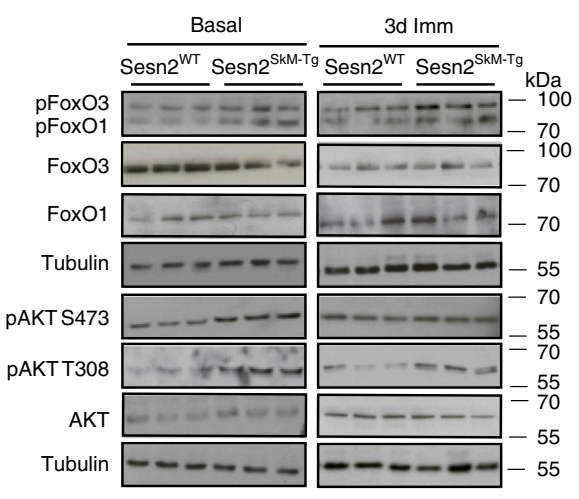

b

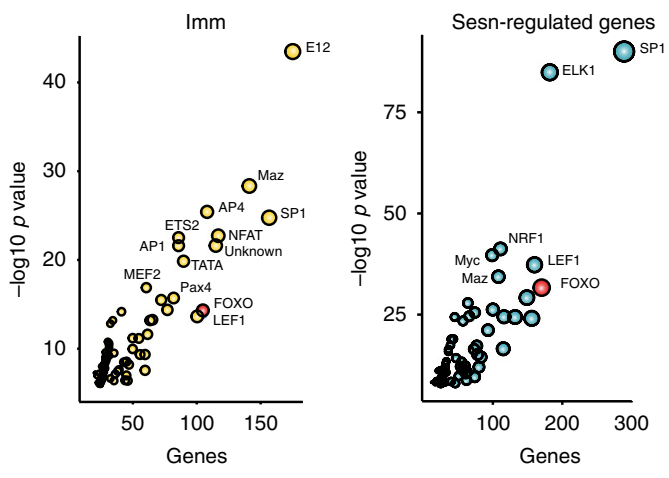

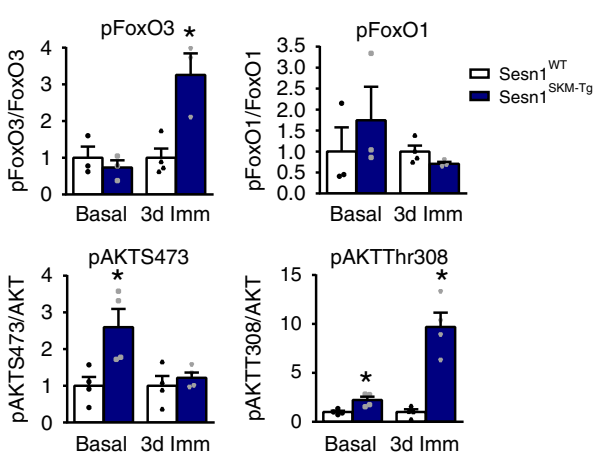
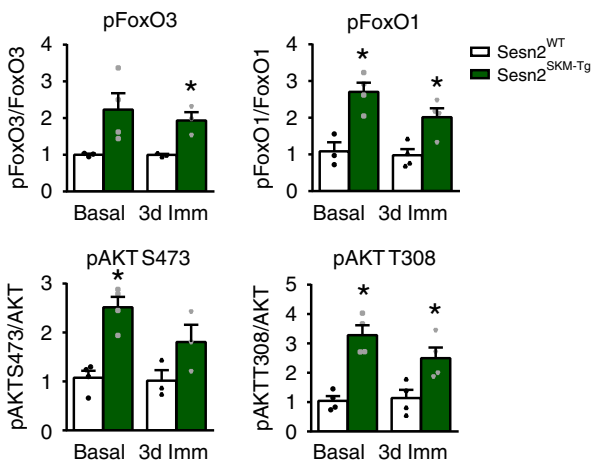

e

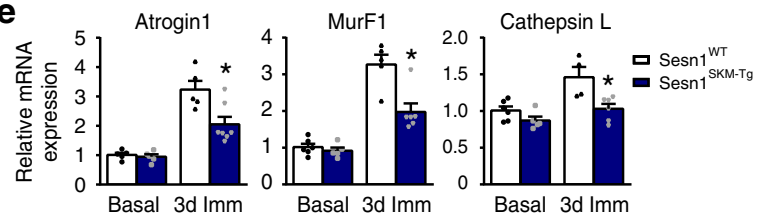

f

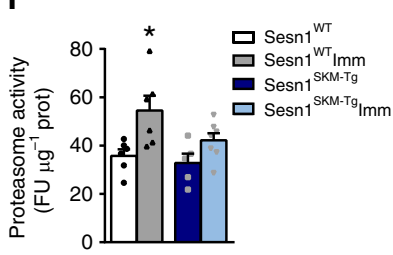

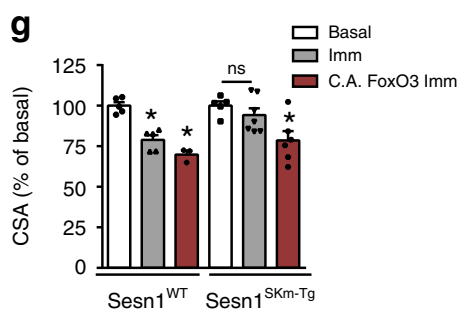

h

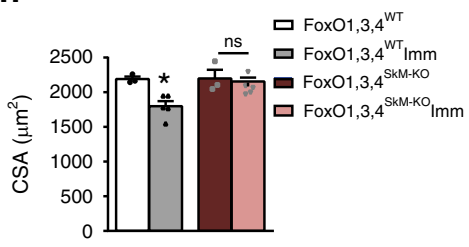

immobilized muscles overexpressing Sesn1 or Sesn2 (Fig. 4c, right panel, and Supplementary Fig. 6c). This observation is consistent with downregulation of mTORC1-dependent ULK1 activation by overexpression of sestrins during immobilization conditions (Fig. 4b). These findings were reinforced by experiments with a tandem fluorescent autophagy flux reporter (mRFP-GFP-LC3 ${ }^{27}$ ) (Fig. 4d). Although mature autolysosomes (red LC3 puncta) were abundant in basal WT muscle, the red LC3 signal was eliminated by muscle immobilization, leaving only yellow puncta (undegraded autophagosomes). Sesn $1 / 2$ overexpression substantially preserved 
Fig. 3 Sestrin blunts FoxO-dependent upregulation of muscle atrogenes. a Gene set enrichment analysis (GSEA) of immobilization-related genes. Bubble plot of enriched GSEA hallmarks in the dysregulated genes upon 3-day muscle immobilization in WT mice (left). Enrichment plots of the combined PI3KAKT-MTOR signaling and mTORC1-signaling hallmarks (AKT-MTORC1 signaling) in comparisons of Sesn1/2 ${ }^{\mathrm{SkM}-\mathrm{Tg}} \mathrm{vs}$. WT mice and Sesn1 ${ }^{\mathrm{KO}}$ vs. WT mice (right). b Bubble plot of enriched transcription factor-binding sites (GSEA) among immobilization-dysregulated genes (left) and the sestrin-regulated gene set defined in Supplementary Fig. 4a (right). c Western blot analysis showing phosphorylation levels of FoxO1, FoxO3, and AKT in muscles of Sesn1WT and

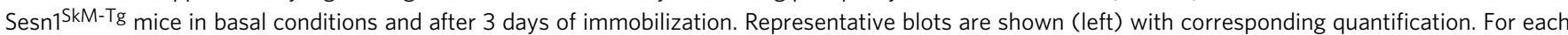
experimental condition (basal and immobilization) values of Sesn $1 \mathrm{SkM}$-Tg are referred to averaged values for Sesn 1 WT samples, which were set to one. d Western blot analysis of the phosphorylation levels of FoxO1, FoxO3, and AKT in muscles of Sesn2 ${ }^{\mathrm{WT}}$ and Sesn2 ${ }^{\mathrm{SkM}-\mathrm{Tg}}$ mice in basal conditions and after 3 days of immobilization. Representative blots are shown (left) with corresponding quantification (right), relatively to Sesn2WT values, as in c. e Atrogin1, MurF1, and Cathepsin L mRNA levels in skeletal muscle from Sesn1WT and Sesn15kM-Tg mice in basal conditions and after 3 days of immobilization. f Proteasome activity in total homogenates of TA muscles from Sesn ${ }^{W T}$ and Sesn1 ${ }^{\mathrm{SkM}}{ }^{\mathrm{Tg}}$ mice in basal conditions and after 3 days of immobilization. g Mean myofiber CSA in TA muscle from Sesn 1 WT and Sesn $1{ }^{\mathrm{SkM}-\mathrm{Tg}}$ mice electrotransferred with control vector or with a plasmid encoding constitutively active $\mathrm{FoxO} 3$ (C.A. FoxO3) and then immobilized for 10 days. Values are relative to basal conditions. $\mathbf{h}$ Mean myofiber CSA in TA muscle from FoxO1,3,4 WT and FoxO1,3,4 $\mathrm{SkM}$-KO mice in basal conditions and after 10 days of immobilization. All data are shown as mean with SEM. Comparisons by Student's $t$-test ( ${ }^{*} p<0.05$ ). Sample numbers were $n=3-4$ mice per group for $\mathbf{c}, \mathbf{d}, n=3-6$ animals for $\mathbf{e}-\mathbf{g}$ and $n=3-5$ mice per genotype and condition for $\mathbf{h}$. Source data are provided as a Source Data file.

the red LC3 puncta during immobilization (Fig. 4d). Conversely, Sesn $1^{\mathrm{KO}}$ mice showed impaired autophagy flux already in basal conditions, with reduced colchicine-dependent LC3-II accumulation (Supplementary Fig. 6d) and loss of red LC3 puncta in the reporter assay (Fig. 4e). The defective autophagy in nonimmobilized muscle of Sesn $1^{\mathrm{KO}}$ mice could be explained by the constitutive mTORC1-dependent ULK1 activation in the absence of sestrin (Supplementary Fig. 6a), since stress-induced sestrin expression inhibits $\mathrm{mTORC1}$ signaling via activation of $\mathrm{AMPK}^{28}$. Consistent with this idea, AMPK activation was readily observed in non-immobilized Sesn1-overexpressing muscle, providing a mechanistic explanation for how sestrin inhibits mTORC1 and activates autophagy (Supplementary Fig. 6e).

We further tested whether sestrin-induced autophagy is important for attenuating disuse atrophy through genetic and pharmacological approaches. Sesn1-mediated protection against disuse atrophy was abolished upon constitutive mTORC1 activation by silencing $\mathrm{TSC} 2$, the negative regulator of mTORC1 ${ }^{29-31}$ (Fig. 4f and Supplementary Fig. 6f). Conversely, rapamycin, which inhibits mTORC1 and induces autophagy ${ }^{32}$, increased WT muscle autophagy flux (Supplementary Fig. 7a), and substantially attenuated muscle atrophy (Fig. 4g), like sestrins. A similar protective effect against muscle atrophy was exerted by the autophagy inducer spermidine $\mathrm{s}^{32,33}$ (Fig. 4h). Autophagy activation through Atg7 overexpression ${ }^{32}$ also protected myofibers from disuse-induced atrophy (Supplementary Fig. 7b, c), whereas muscle-specific genetic deletion of Atg7 (Atg7 ${ }^{\text {SkM-KO}) ~(S u p p l e m e n t a r y ~ F i g . ~ 7 d) ~ n u l l i f i e d ~ S e s n 1-m e d i a t e d ~}$ protection against disuse-induced muscle atrophy (Fig. 4i). No significant crosstalk between both catabolic activities was observed during muscle immobilization (not shown). Taken together, these data demonstrate that muscle inactivity produces detrimental effects by differentially acting on catabolic mechanisms (i.e. decreasing autophagic while inducing proteasomal pathways). By reversing this concert, sestrins preserve autophagy that is essential for muscle homeostasis, while preventing proteasome overactivation that wastes muscle by majorly driving loss of muscle mass during disuse condition.

Sestrins protect muscle against aging-associated atrophy. We finally investigated whether sestrins can also protect against agingassociated muscle atrophy (sarcopenia). Compared with young mice ( 4 months old), aged mice ( 24 months old) showed lower expression of Sesn1 protein in skeletal muscle (Fig. 5a) accompanied by pronounced loss of skeletal muscle force and mass and myofiber atrophy (Fig. 5b-e). All these muscle parameters were improved by transduction of AAV-Sesn 1 for one month, including a slight reduction in muscle fibrosis (Fig. 5c-e and Supplementary Fig. 7e), suggesting that sestrin activation presents a promising strategy for protecting against age-associated muscle atrophy and related conditions. No changes in myonuclear number were observed (Supplementary Fig. 7f). Mechanistically, we found that mTORC1 activity is increased in muscles of aged mice and is reduced by Sestrin overexpression (Fig. 5f), correlating with the protection exerted by Sestrin on age-related muscle atrophy.

Finally, we compared publicly available transcriptomic data of skeletal muscles of young and old mice (GEO: GSE53959) ${ }^{34}$ to assess for potential gene expression similarities between aged and immobilized muscles. Interestingly, we found that aged-muscle transcriptome was enriched in gene expression signatures for apoptosis, inflammation, cell-cycle inhibition, UV response, Myc targets, and anabolic signaling (PI3K/AKT/mTORC1 regulation) (Fig. 5g). Supporting the existence of commonalities between genes induced by muscle immobilization and aging, the aged muscle transcriptome was found to be also enriched in atrogenes (Fig. 5h), while the upregulated genes contained binding sites for E12, Myc/Maz, Sp1, and FoxO TFs (Fig. 5i). In fact, the transcriptome of old mice was enriched in FoxO and Sestrinregulated atrogenes ${ }^{24}$, including the E3 ubiquitin-ligases Atrogin1 and Musa1 (Fbxo30) ${ }^{23}$ and autophagy-related genes Bnip3 and p62 (Sqstm1) (Fig. 5i). Taken together, these results suggest that, similar to disuse-induce muscle atrophy, sestrins may protect muscle against aging-associated muscle atrophy by coordinating anabolic and catabolic pathways.

\section{Discussion}

Autophagy and ubiquitin-proteasome proteolytic activities have been independently linked to muscle wasting $6,31,35$. Our analysis combining unbiased transcriptomics and targeted transgenesis approaches has identified an important mechanism for protecting muscle from wasting during disuse and aging. Sestrins, strongly downregulated by disuse, preserve muscle mass by coordinately inhibiting atrophic proteolysis and activating homeostatic autophagy. Sestrins may achieve disuse-induced protection by regulating the balance between distinct growth-associated mTOR complexes, strongly inhibiting mTORC1 ${ }^{28}$ while supporting mTORC2 activity ${ }^{14}$. Through upregulation of AKT, sestrins inhibit FoxO-dependent transcription of atrogenes, which normally promote muscle wasting by accelerating protein degradation. At the same time, through the activation of AMPK and inhibition of $\mathrm{mTORC}^{28}$, sestrins upregulate autophagy, thus maintaining proteostasis and organelle quality for homeostatic preservation of muscle mass and force. Of interest, the protective effect of sestrin was also extended to denervation-induced muscle 


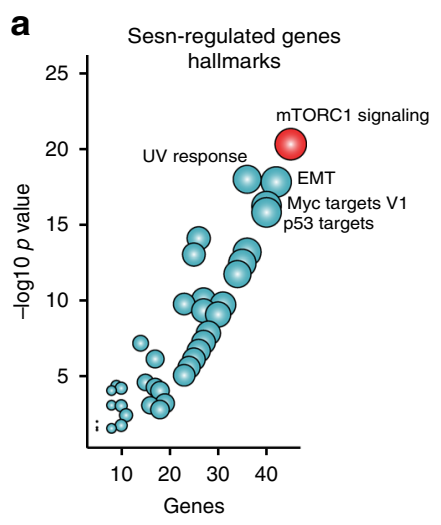

b
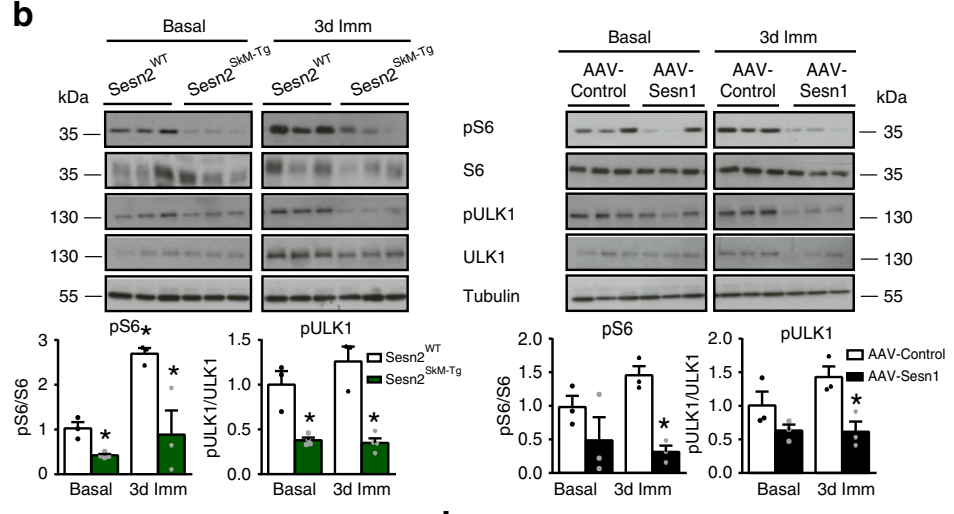

C
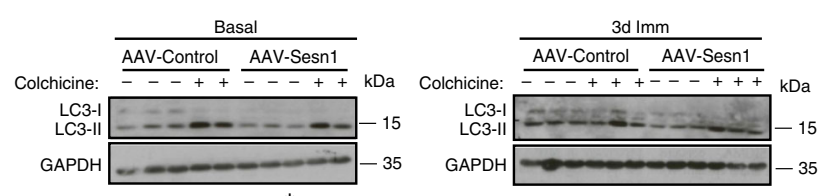

d
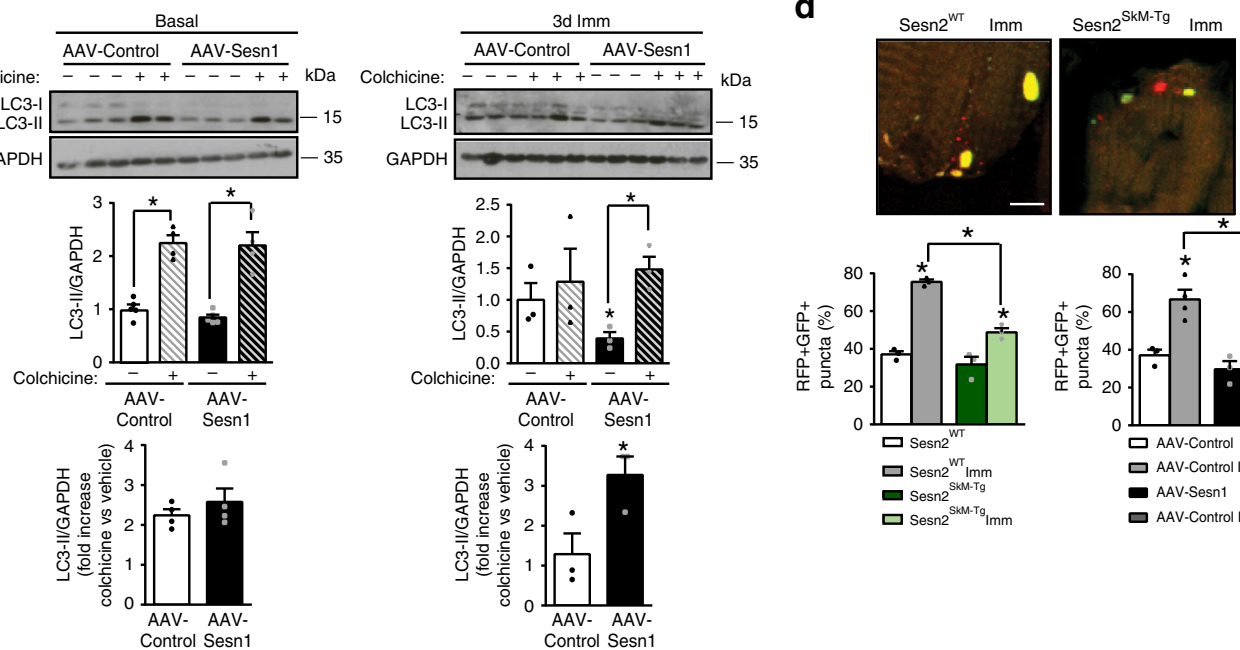

e

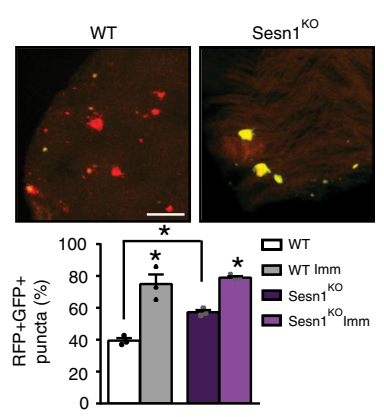

h
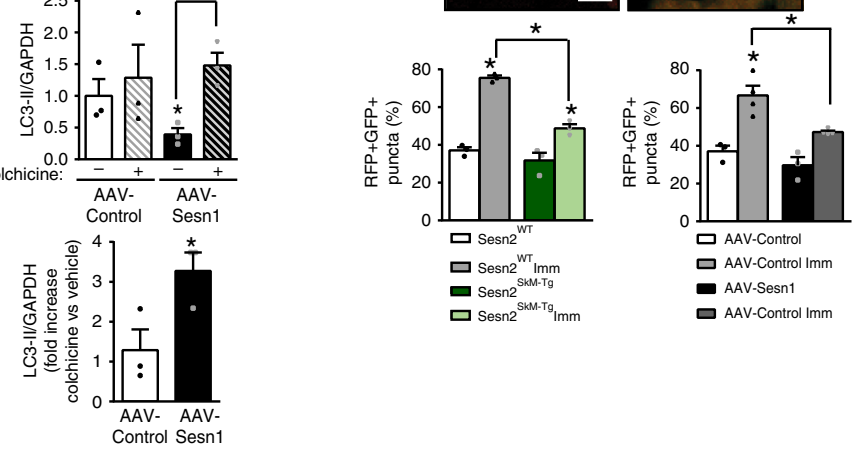

$\mathbf{f}$

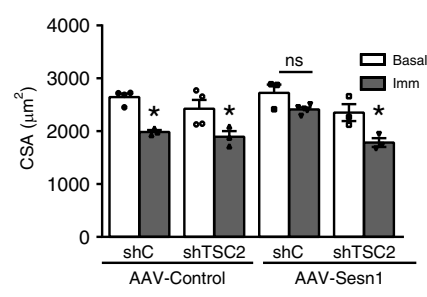

\section{g}

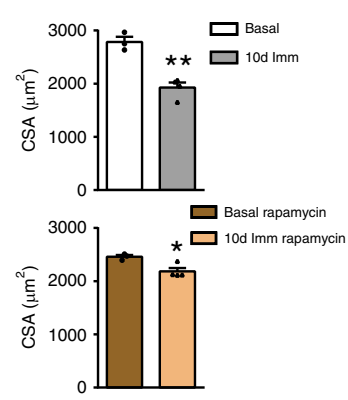

i

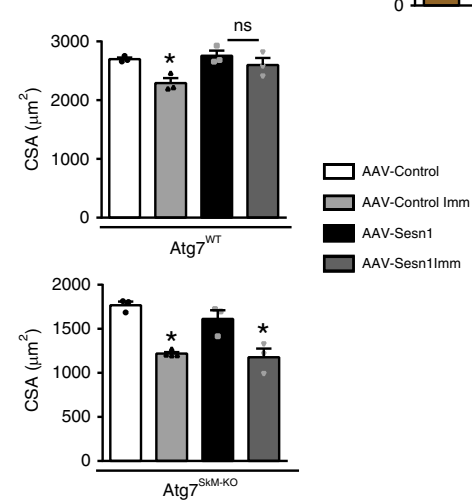

muscle mass and force significantly affects health, life quality, and even survival. Given their beneficial effects on muscle wasting during disuse and aging, sestrins should be regarded as nodal regulators of mammalian muscle growth with potentially broad applications in the treatment of common catabolic conditions. wasting and age-associated muscle atrophy. The relevance of our data is further supported by recent findings demonstrating reduction of sestrins' protein levels in muscles of aging human individuals ${ }^{36}$ including muscles from the frail elderly popula$\operatorname{tion}^{37}$. Future studies should explore the effect of Sestrin/mTOR modulators such as NV-5138 $8^{38}$ on these conditions. Loss of 
Fig. 4 Autophagy induction via sestrin-mediated mTORC1 blockade prevents atrophy. a Bubble plot showing the main molecular hallmarks (GSEA) enriched in the sestrin-regulated gene set defined in Supplementary Fig. 4a. b Western blot analysis and quantification of S6 and ULK1 phosphorylation in muscles from Sesn $2^{\mathrm{WT}}$ and Sesn2 ${ }^{\mathrm{SkM}}$-Tg mice (left) and in muscles transduced with AAV-Sesn1 or AAV-Control (right) in basal conditions and at 3 days post-immobilization. Values were normalized to basal control conditions. c Western blot analysis and quantification of LC3I and LC3II in TA muscles transduced with AAV-Sesn1 or AAV-Control in basal conditions and at 3 days post-immobilization. Treatment of mice with colchicine or vehicle is indicated. Lower chart shows the fold increase in LC3II content in colchicine-treated versus vehicle-treated mice. $\mathbf{d}$ Representative confocal images of 3-day-immobilized TA muscle electrotransferred with a tandem mRFP-GFP-LC3 reporter plasmid to enable detection of autophagosomes (yellow puncta) and autolysosomes (red puncta). The chart shows double RFP ${ }^{+}$GFP + puncta as a percentage of total puncta for the indicated genotypes. Scale bar $=5 \mu$ m. e Representative confocal images of non-immobilized muscles in WT and Sesn $1^{\mathrm{KO}}$ mice treated as in d. The chart shows RFP+GFP+ puncta as a percentage of total puncta. Scale bar $=5 \mu \mathrm{m}$. $\mathbf{f}$ Mean TA myofiber CSA in basal conditions and at 10 days post-immobilization in muscles transduced with AAV-Control or AAV-Sesn1 and electrotransferred with control short hairpin (sh) or sh targeting TSC2. g Mean TA myofiber CSA in basal conditions and at 10 days post-immobilization in WT mice treated with vehicle (top) or rapamycin (bottom). h Mean TA myofiber CSA in basal conditions and at 10 days post-immobilization in WT mice treated with vehicle or spermidine. i Mean TA myofiber CSA in basal conditions and at 10 days post-immobilization in Atg 7 WT and Atg7 ${ }^{S k M-K O}$ mice transduced with AAV-Control or AAV-hSesn1. All data are shown as mean with SEM. Comparisons by Student's $t$-test ( ${ }^{*} p<$ 0.05 and ${ }^{* \star} p<0.01$ vs. basal). Sample numbers were $n=3$ mice per group for $\mathbf{b}, \mathbf{d}, \mathbf{e}, n=3-5$ animals for $\mathbf{c}, \mathbf{f}, \mathbf{g}, \mathbf{i}$ and $n=4$ for $\mathbf{h}$. Source data are provided as a Source Data file.

\section{Methods}

Animals. The different mouse models used in this study were generated as follows:

The skeletal muscle-specific Sesn 1 transgenic mouse model (Sesn 1 SkM-Tg) was generated in C57BL/6 background and carry a transgene coding for human Sesn1 under the control of MCK promoter. The human Sesn1 cDNA sequence (from LV-Sesn $1^{19}$ ) was subcloned into the pBluescript-MCK plasmid (a kind gift from Markus Rüegg). A $4 \mathrm{~kb}$ PacI digestion fragment was excised, microdialyzed, and microinjected into the pronuclei of fertilized mouse eggs $(\mathrm{C} 57 \mathrm{BL} / 6 \mathrm{~J} \times \mathrm{C} 57 \mathrm{BL} / 6 \mathrm{~J})$ at the Mouse Mutant Core Facility, Institute for Research in Biomedicine (Barcelona, Spain). Embryos were implanted into pseudo-pregnant foster females (ICR), and transgenic pups were identified. DNA samples from tail clips of subsequent litters were screened by PCR with primers spanning different sequences of MCK promoter and Sesn 1 cDNA (Primer set 1 forward CTGCCCCCGGGTCACCACC reverse TCGATTCAGGTCATATAGCGGGT; Primer set 2 forward CAGGGCTTATAC GTGCCTGGGACTC reverse TGTGGGTGGAAAACCATCACTAACG; Primer set 3 forward GCCCCCGGGTCACCACCAAG reverse GGCCAAGCGCATGGATCC TTTTA) that amplified a 1550,600 , and $76 \mathrm{bp}$ fragments, respectively. The transgene was maintained on the C57BL/6J background throughout the study.

The skeletal muscle-specific Sesn2 transgenic mouse model (Sesn $2^{\mathrm{SkM}-\mathrm{Tg}}$ ) was generated by crossing mice that express Sesn 2 from a Tet-regulated promoter (provided by Dr. M. Karin, UCSD, San Diego, USA) with mice harboring the skeletal muscle-specific MCK-tTA construct (obtained from Dr. M. Ruegg, Biozentrum, Basel, Switzerland). Non-transgenic littermates (that were WT for Sesn 1 and Sesn 2 expressions) were used as controls for the two transgenic mouse lines, and named Sesn $1^{\mathrm{WT}}$ and Sesn $2^{\mathrm{WT}}$ mice, respectively. Sesn 1 KO mice were provided by Dr. J.H. Lee (University of Michigan, USA) and analyzed in comparison with normal, control WT mice. Atg7 ${ }^{\text {SkM-KO }}$ mice were generated as previously described ${ }^{32}$, and FoxO1,3,4 $4^{\text {KkM-KO }}$ mice were obtained by crossing FoxO1/3/4-floxed mice (a kind gift from Dr. M. Sandri, University of Padova, Italy) with the Pax 7 Cre line (provided by Dr. M. Capecchi, University of Utah, USA). Non-transgenic littermates (that were WT for FoxO1,3,4 and for Atg7 expression) were used as controls for Atg7 ${ }^{\mathrm{SkM}-\mathrm{KO}}$ mice and for FoxO1,3,4 $\mathrm{SkM}-\mathrm{KO}$ mice, and named Atg7 WT and FoxO1,3,4 ${ }^{\mathrm{WT}}$ mice, respectively. Therefore, Sesn 1 WT, Sesn 2 WT, WT, Atg7 ${ }^{\mathrm{WT}}$, and FoxO1,3,4 ${ }^{\mathrm{WT}}$ mice are all equally WT, non-transgenic mice, but each one is used as littermate control of each individual genetically modified mouse line.

Mice were housed in standard cages under $12 \mathrm{~h}$ light/dark cycles with ad libitum access to food and water. All experiments were performed on 4-5-monthold male mice. All animal experiments were approved by the Ethics Committee of the Barcelona Biomedical Research Park (PRBB) and performed according to Catalan and European legislation.

Induction of muscle atrophy. The immobilization protocol was performed unilaterally on anesthetized animals as formerly described ${ }^{39}$. Briefly, the right hindlimb was immobilized with rigid plastic sticks fixed with a medical adhesive bandage. This procedure prevented movement of the immobilized leg alone. Denervation was performed as previously described ${ }^{40}$. In brief, a $5 \mathrm{~mm}$ segment of the sciatic nerve was surgically removed down to the gluteus maximum from the right leg. Mice not subjected to atrophy-promoting conditions were used as controls (Basal). Muscles were removed at 3 or 10 days after atrophy induction and frozen in liquid nitrogen for subsequent analyses.

In vivo gene electrotransfer and AAV injection. Expression plasmids used for electrotransfer studies were purified using a Endofree plasmid kit (Qiagen) and dissolved in $0.9 \% \mathrm{NaCl} .45 \mathrm{~min}$ before electrotransfer, muscles were pretreated with hyaluronidase ( $10 \mathrm{U} /$ muscle). Afterwards, naked plasmids ( $60 \mu \mathrm{g}$ DNA) were injected into the tibialis anterior muscle and 10 pulses of $20 \mathrm{~ms}$ each were applied to each hindlimb at $175 \mathrm{~V} / \mathrm{cm}$ and $1 \mathrm{~Hz}$ using an electroporator (ECM 830; BTX). Empty vector was used as control.

AAV for in vivo expression of Sesn 1 and Sesn2 were generated and provided by the Virus Production Unit (UPV, UAB, Barcelona). AAVs were diluted in $0.9 \%$ $\mathrm{NaCl}$ at $0.25^{\star} 10^{13} \mathrm{gc} / \mathrm{ml}$ and directly injected into muscles $(40 \mu \mathrm{l} / \mathrm{TA}$ and $10 \mu \mathrm{l} / \mathrm{EDL}$ and soleus muscles). AAV-GFP was used as a control. Four days after transduction mice were subjected to the immobilization protocol.

Pharmacological treatments. Mice were injected with rapamycin $(4 \mathrm{mg} / \mathrm{kg}$ body weight) (which induces autophagy) or vehicle (DMSO) intraperitoneally (i.p.) every other day for 2 weeks. Colchicine (which inhibits autophagy) was injected i.p. $\left(0.4 \mathrm{mg} / \mathrm{kg}^{*}\right.$ day) 2 days before sacrifice. The proteasome inhibitor Bortezomib $(0.1 \mathrm{mg} / \mathrm{kg})$ or vehicle (DMSO) was injected i.p. every other day for 2 weeks. Mice were treated with $3 \mathrm{mM}$ spermidine in drinking water for 2 weeks.

Muscle force measurement. Ex vivo force measurements of EDL and soleus muscles was assessed as previously described ${ }^{41}$. Briefly, mice were sacrificed, and muscles were immediately excised and placed into a dish containing oxygenated Krebs-Henseleit solution. Muscles were mounted vertically in a temperature controlled $\left(30^{\circ} \mathrm{C}\right)$ chamber and immersed in the Krebs-Ringer bicarbonate buffer solution, with $10 \mathrm{mM}$ glucose, also continuously oxygenated. One end of the muscle was linked to a fixed clamp, while the other end was connected to the leverarm of an Aurora Scientific Instruments 300B actuator/transducer system, using a nylon thread. The optimum muscle length (Lo) was determined from micromanipulations of muscle length to produce the maximum isometric twitch force. Maximum isometric-specific tetanic force was determined from the plateau of the curve of the relationship between specific isometric force with a stimulation frequency ranging from 1 to $200 \mathrm{~Hz}$. Force was normalized per muscle area (determined by dividing the muscle mass by the product of longitude and the density of muscle $\left.\left(1.06 \mathrm{mg} / \mathrm{mm}^{3}\right)\right)$ to calculate the specific force $\left(\mathrm{mN} / \mathrm{mm}^{2}\right)$.

Muscle histology and immunohistochemistry. Muscles were embedded in OCT solution (TissueTek), frozen in isopentane cooled with liquid nitrogen and stored at $-80^{\circ} \mathrm{C}$ until analysis. $10 \mu \mathrm{m}$ muscle cryosections were collected and stained for hematoxylin/eosin (H/E) or Sirius red (Sigma-Aldrich).

For immunohistochemistry assays, muscle cryosections were examined by standard immunohistochemical procedures for the expression of myosin heavy chain (MHC) isoforms. The primary monoclonal antibodies employed were antimyosin I (A4.840), anti-myosin IIA (A4.74), and anti-myosin IIB (BF-F3) (Developmental Studies Hybridoma Bank).

Digital images were acquired using the Leica DMR600B microscope equipped with a DFC300FX camera. Fiber type distribution, CSA, and percentage of muscle area positive for Sirius red staining were quantified using Image J software, as previously reported ${ }^{42,43}$.

For myonuclei quantification, muscle sections were immunostained for dystrophin (1/400), the secondary antibody was coupled to Alexa-488 and nuclei were stained with DAPI (Invitrogen). Images were acquired using a Leica TCS SP5 confocal scanning microscope system.

Fluorescence microscopy analysis of muscle sections. TA muscles were removed, fixed in PFA $2 \%$ for $4 \mathrm{~h}$ at $4{ }^{\circ} \mathrm{C}$ and incubated with $15 \%$ sucrose overnight at $4{ }^{\circ} \mathrm{C}$. Then, muscles were embedded in OCT solution (TissueTek), immediately frozen in liquid nitrogen-cooled isopentane and stored at $-80^{\circ} \mathrm{C} .10 \mu \mathrm{m}$ cryosections of TA muscle (which has been transfected with mRFP-GFP-LC ${ }^{44}$ ) were analyzed using a Leica TCS SP5 confocal scanning microscope system. Colocalization of RFP-LC3 and GFP-LC3 puncta was determined on the maximum 
a
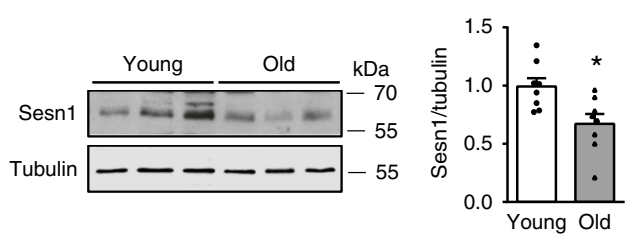

C
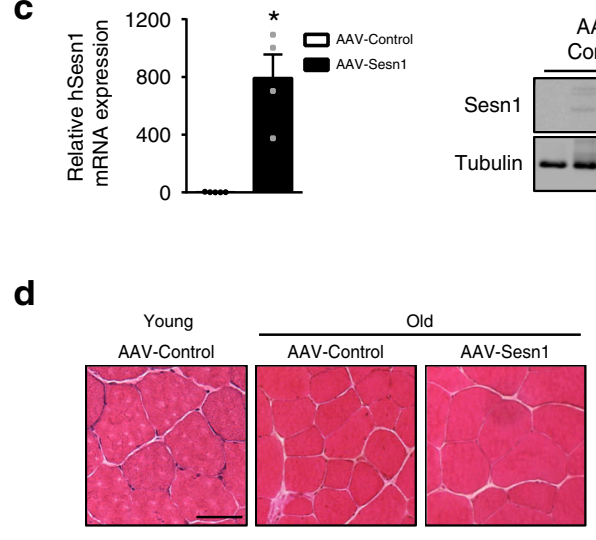
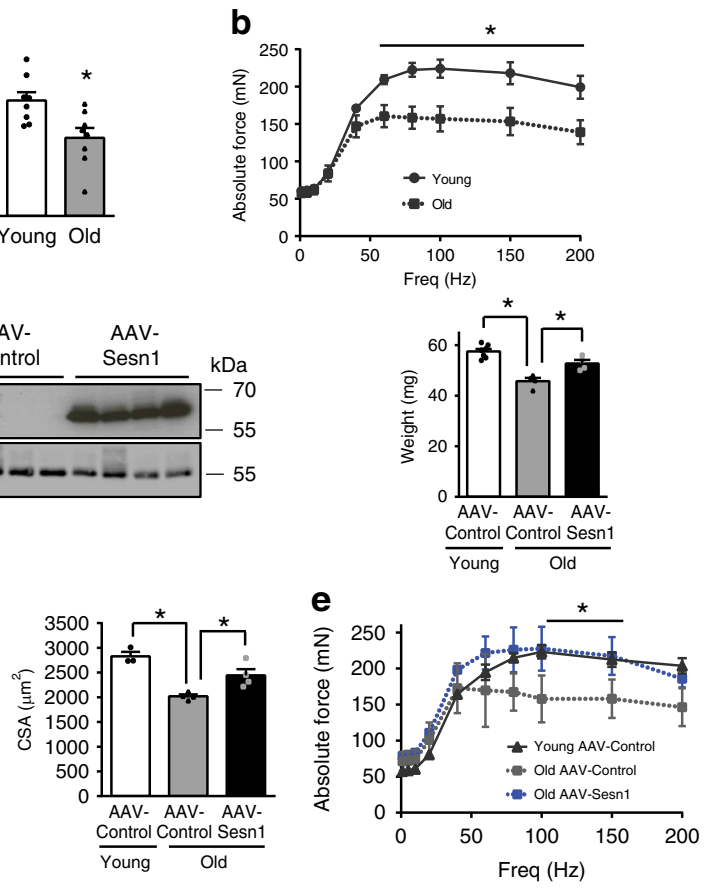

$\mathbf{f}$
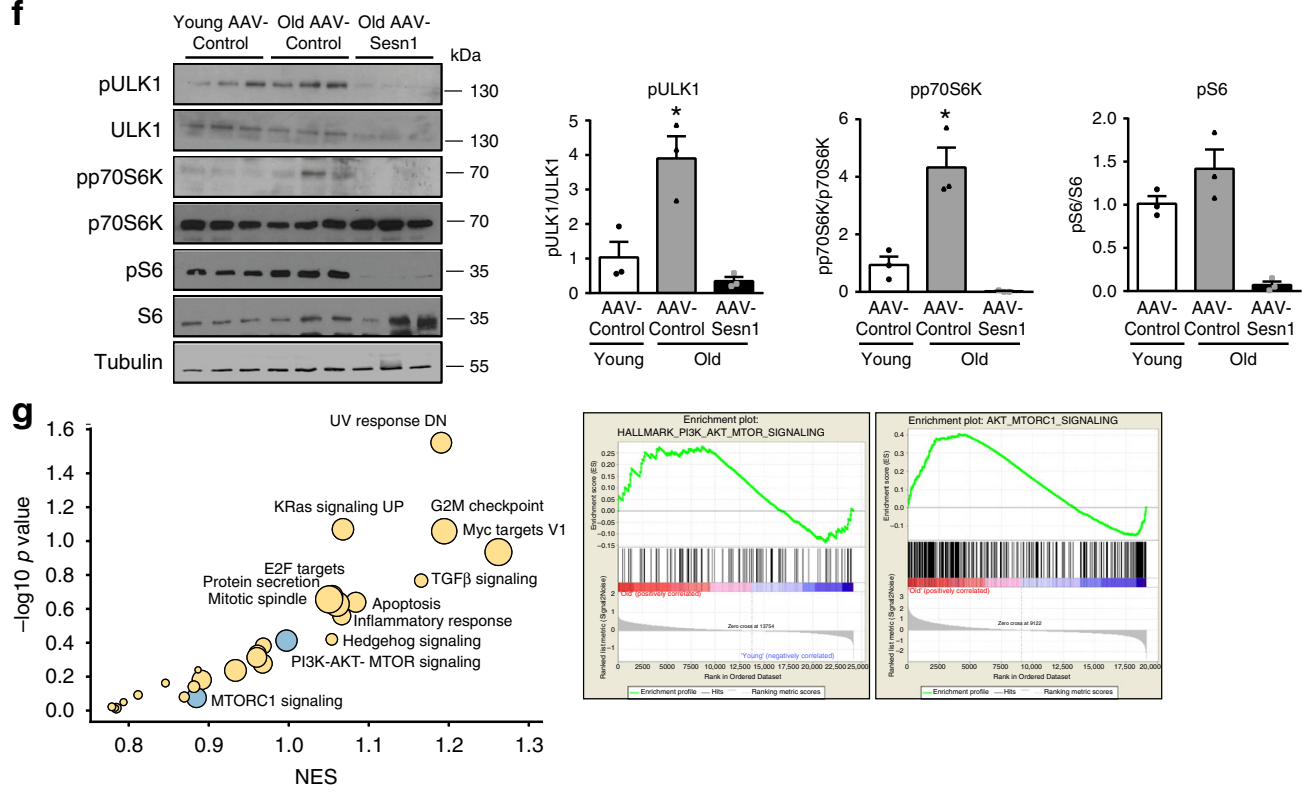

h
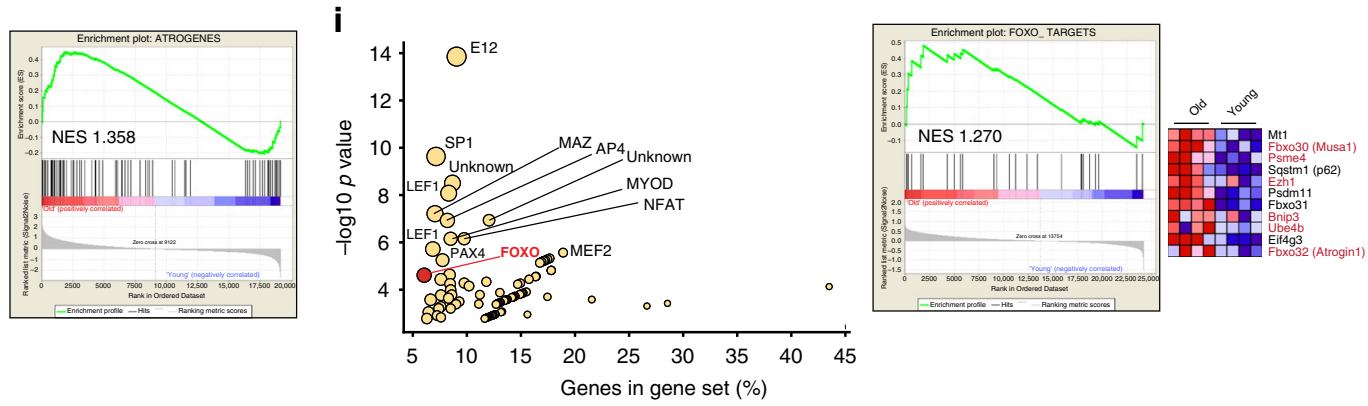

projection of $10-\mathrm{z}$ sections. Note: Measuring autophagy flux through this method is based on the concept of lysosomal quenching of GFP. GFP is a stably folded protein and relatively resistant to lysosomal proteases. However, the low $\mathrm{pH}$ inside the lysosome quenches the fluorescent signal of GFP, which makes it difficult to trace the delivery of GFP-LC3 to lysosomes. In contrast, RFP exhibits more stable fluorescence in acidic compartments, and mRFP-LC3 can be readily detected in autolysosomes. By exploiting the difference in the nature of these two fluorescent proteins (that is, lysosomal quenching of GFP fluorescence versus lysosomal stability of RFP fluorescence), autophagic flux can be morphologically traced with an mRFP-GFP-LC3 tandem construct. With this tandem construct, autophagosomes and autolysosomes are labeled with yellow (mRFP and GFP) and red (mRFP only) signals, respectively. 
Fig. 5 Sestrins prevent aging-related muscle atrophy. a Western blot and quantification of Sesn1 protein levels in skeletal muscle from young (4 months) and old (24 months) mice. b Force-frequency curve of EDL muscles from young and old WT mice. c qPCR of human Sesn1 mRNA in skeletal muscle from 24-month-old mice transduced with AAV-Sesn1 for one month and Sesn1 protein expression by Western blotting in the same muscles (left). Weight of TA muscles from young and old mice transduced with AAV-Sesn1 or AAV-Control for 1 month (right). d Representative H/E staining pictures of TA muscle sections from 24-month-old mice transduced with AAV-Sesn1 or AAV-Control for 1 month and quantification of mean myofiber CSA. Scale bar $=50 \mu$ m. e EDL force-frequency curve in 24-month-old mice transduced with AAV-Sesn1 or AAV-Control for 1 month. $\mathbf{f}$ Western blot analysis and quantification of phosphorylation levels of p70S6K, S6, and ULK1 in muscles from old mice transduced with AAV-Sesn1 or AAV-Control for 1 month. Representative blots are shown with corresponding quantification. Values were normalized to averaged values of young control mice. $\mathbf{g}$ Bubble plot of enriched GSEA hallmarks in the dysregulated genes upon muscle aging (left). Enrichment plots of the PI3K-AKT-MTOR signaling hallmark and the combined PI3K-AKT-MTOR signaling and mTORC1 signaling hallmarks (AKT-MTORC1 signaling) in comparisons of old and young mice (right). $\mathbf{h}$ As in $\mathbf{g}$, enrichment plots of the atrogenes gene set in comparisons of old and young mice. i Bubble plot of enriched transcription factor-binding sites (GSEA) in the dysregulated genes upon muscle aging (left). Enrichment plots of the FoxO3a targets in skeletal muscle (as defined in Brocca et al. ${ }^{24}$ ) and heatmap illustrating genes with higher enrichment in comparisons of old and young mice (right). All data are shown as mean with SEM. Comparisons by Student's $t$-test $\left({ }^{*} p<0.05\right)$. Sample numbers were $n=8$ mice per group for $\mathbf{a}, n=4-7$ mice per condition for $\mathbf{b}$ and $\mathbf{c}, n=3-5$ mice per condition for $\mathbf{d}$ and $\mathbf{e}$ and $n=3$ mice per group for $\mathbf{f}$. Source data are provided as a Source Data file.

Table 1 Primers used for qPCR.

\begin{tabular}{llll} 
Gene & Species & Forward primer & Reverse primer \\
\hline Sesn1 & Mouse & GTCTGGATAACATCACATTAG & CCAGGTAGGAACACTGATGC \\
Sesn2 & Human & CAGCATTGGAAACATTAGGCAA & CCGAGACTCGGTATTTGAAAGC \\
Sesn3 & Mouse & TAGCCTGCAGCCTCACCTAT & TATCTGATGCCAAAGACGCA \\
Fbxo32 & Mouse & GGCAAATCTTCGTACCCAA \\
Trim63 & Mouse & CATGCGTCCTCACTCAGA & CACCCACATGTTAATGTTGCCC \\
Ctsl & Mouse & AAGGCTGTTGGAGCTGATAGCA & AAACGACCTCCAGACATGGACA \\
FoxO3 & Mouse & TGCCTGGAGATGTTACCAAGC & TCCGTCCTTCGCTTCATAGG \\
FoxO4 & Mouse & GTGGACTGTTCTCACGCTCAAG & GGGGTGAGGGCATCTTT \\
Atg7 & CTGGG & CTGGGTCAGGCTAAGAGT & TCATTCTGAACGCGCATGAAG \\
L7 & Mouse & CTGGGGAACCTGTCCTATG & ACAGGATCGGTTCGGAGTGT \\
\end{tabular}

RNA isolation, reverse transcription, and quantitative PCR. Total RNA from TA muscles was isolated with QIAzol Lysis Reagent (Qiagen) and quantified with Nanodrop. M-MLV Reverse Transcriptase (Promega) was used to synthesize cDNAs from $1 \mu \mathrm{g}$ total RNA following the manufacturer's instructions. RT-qPCR reactions were performed with SYBR Green in 384-well plates using the Roche LC480 cycler (Roche Applied Science). All data were normalized to L7 expression. Primer sequences are listed in Table 1.

Transcriptomic analysis. For RNAseq analysis, total RNA from TA muscles was extracted using a protocol combining QIAzol Lysis Reagent and RNAeasy minikit columns (Qiagen) following the manufacturer's instructions. RNAseq services were provided by the CNIC Genomics Unit, including quality control tests of total RNA using Agilent Bioanalyzer and Nanodrop spectrophotometry. cDNA library preparation and amplification were performed from $200 \mathrm{ng}$ total RNA using NEBNext Ultra RNA Library Prep Kit for Illumina. RNAseq analysis was performed with 3-4 samples per condition, using Illumina Hiseq 2500. Sequencing reads were preprocessed by means of a pipeline that used FastQC, to asses read quality, and Cutadapt 1.7.1 to trim sequencing reads, eliminating Illumina adaptor remains, and to discard reads that were shorter than $30 \mathrm{bp}$. The resulting reads were mapped against the mouse transcriptome (GRCm38, release 76; aug2014 archive) and quantified using RSEM v1.2.20. Data were then processed with a differential expression analysis pipeline that used Bioconductor package LIMMA for normalization and differential expression testing. For differential expression analysis we filtered for genes that showed at least $\log 2 \mathrm{FC} 0.25(\geq+0.25$ for upregulation; and $\leq-0.25$ for downregulation) and an adj. $p$ value $<0.05$.

FoxO promoter-reporter assay. TA muscles from Sesn $1^{\mathrm{WT}}$ and Sesn $1^{\mathrm{SkM}-\mathrm{Tg}}$ mice were electrotransferred with a reporter plasmid with three copies of forkhead responsive element linked to the luciferase reporter gene (FHRE-luciferase; Addgene). After 3 days of immobilization, muscles were collected and luciferase activity was measured in muscle homogenates by using Dual-Luciferase Reporter Assay Kit (Promega Corporation, USA). Values were normalized to nonimmobilized muscles.

Muscle protein extraction and Western blotting. Total homogenates from skeletal muscle were obtained in IP buffer $(50 \mathrm{mM}$ Tris- $\mathrm{HCl}$ pH 7.5, $150 \mathrm{mM} \mathrm{NaCl}$, $1 \%$ NP-40, 5 mM EGTA, $5 \mathrm{mM}$ EDTA, $20 \mathrm{mM} \mathrm{NaF}, 25 \mathrm{mM}$-glycerophosphate,
$0.1 \mathrm{mM}$ sodium vanadate, $1 \mathrm{mM}$ PMSF) supplemented with protease and phosphatase inhibitors (Complete Mini, Roche Diagnostic Corporation; phosphatase inhibitor cocktail, Sigma). Protein concentration was measured using the Bradford method (Protein Assay, Bio-Rad). $40 \mu \mathrm{g}$ of protein were resolved by SDS-PAGE and transferred to PVDF membranes (Millipore). Membranes were blocked with $5 \%$ milk in TBS-T for $1 \mathrm{~h}$ and incubated with primary antibodies overnight at $4{ }^{\circ} \mathrm{C}$ in $5 \%$ BSA in TBS-T (Tubulin 1:4000 and others 1:1000). Proteins were detected by the ECL method and quantified by scanning densitometry. The antibodies used are listed in Table 2. Uncropped versions of all blots shown in figures are supplied in the Source Data File.

Proteasome activity in muscle. Proteasome activity in total homogenates from TA muscles was determined by evaluating the cleavage of specific fluorogenic substrates. Muscles were homogenized in lysis buffer ( $50 \mathrm{mM}$ Tris- $\mathrm{HCl} \mathrm{pH} 7.5$, $250 \mathrm{mM}$ Sucrose, $5 \mathrm{mM} \mathrm{MgCl}_{2}$, $0.5 \mathrm{mM}$ EDTA, $2 \mathrm{mM} \mathrm{ATP}$, and $1 \mathrm{mM}$ DTT) and centrifuged at $12,000 \times g$ for $30 \mathrm{~min}$ at $4{ }^{\circ} \mathrm{C}$. The supernatant was collected and protein concentration determined by the method of Bradford. For chymotrypsinlike activity, aliquots of $20 \mu \mathrm{g}$ protein were incubated for $60 \mathrm{~min}$ at $37^{\circ} \mathrm{C}$ in the presence of $100 \mu \mathrm{M}$ of the fluorogenic substrate succinyl-Leu-Leu-Val-Tyr-7amino-4-methylcoumarin (Suc LLVY-AMC). Each assay was conducted in the absence and presence of the specific proteasomal inhibitor MG132 (Sigma-Aldrich) at $20 \mu \mathrm{M}$. Fluorescence was read with a spectrofluorometer (390 nm excitation/ $460 \mathrm{~nm}$ emission; Tecan Infinite M200). The activity was expressed as units of fluorescence per microgram of protein, as a percentage of the control group. All samples were assayed in triplicate using at least four animals.

Bioinformatic analysis. Hierarchical clustering of expression values (after filtering expression values below one in all the samples) was carried out with Morpheus (https://software.broadinstitute.org/morpheus/) using one minus Pearson correlation with a complete linkage. GSEA of Sestrin-regulated genes was performed using GSEA web interface with the Molecular Signatures Database "hallmarks" and "transcription factor binding targets" genesets, to reveal pathways and cisregulatory motifs which can function as potential transcription factor-binding sites, respectively ${ }^{45}$. The Java implementation code from the Broad Institute was used for direct GSEA comparisons of the raw data from our RNA-seq experiments or with GEO: GSE5395 data set with the "AKT-MTOR signaling" gene set (generated by combining the "MTORC1 Signaling" and the "PI3K-AKT- MTOR signaling" 
Table 2 Antibodies.

\section{Antibody}

Rabbit anti-Sesn

Rabbit anti-Sesn2

Rabbit anti-phospho-AKT (Ser473)

Rabbit anti-phospho-AKT (Trh308)

Mouse anti-AKT (pan)

Rabbit anti-phospho-FoxO1 (Thr24)/FoxO3a (Thr32)

Rabbit anti-phospho-S6 (Ser235/236)

Mouse anti-S6

Anti-Phospho-AMPK $\alpha$ (Thr172)

Anti-AMPK $\alpha$

Rabbit anti-phospho-ULK1

Rabbit anti-ULK1

Rabbit anti-LC3B

Mouse anti-Tubulin

Mouse anti-GAPDH

\section{Source}

Reference

Provided by Dr. Jun Hee Lee

Proteintech

Cell Signaling Technology

Cell Signaling Technology

Cell Signaling Technology

Cell Signaling Technology

Cell Signaling Technology

Cell Signaling Technology

Cell Signaling Technology

Cell Signaling Technology

Cell Signaling Technology

Cell Signaling Technology

Cell Signaling Technology

Sigma Aldrich

Santa Cruz Biotechnology
Cat\#10795-1-AP

Cat\#9271

Cat\#4056

Cat\#2920

Cat\#9464

Cat\#4858

Cat\#2317

Cat\#2531

Cat\#2532

Cat\#14202

Cat\#8054

Cat\#2775

Cat\#T-6199

Cat\#sc-32233
Molecular Signatures Database hallmarks), the growth and atrophy regulators gene set (assembled by combining the following Gene Ontology categories: positive and negative regulations of insulin-like growth factor receptor signaling pathway, positive and negative regulations of TORC1 signaling and positive and negative regulations of muscle atrophy) and the atrogenes gene set (that was manually curated from selected bibliography $\left.{ }^{1-4,6,7,22,24}\right)$. Bubble plots were generated using ggplot2 library in R or Seaborn in Python. Venn diagrams were generated using The BEG Ugent tool (http://bioinformatics.psb.ugent.be/webtools/Venn/).

Statistical analysis. For mouse experiments, no specific blinding method was used, but mice in each sample group were selected randomly. The sample size $(n)$ of each experimental group is described in each corresponding figure legend.

GraphPad Prism software was used for all statistical analyses. Quantitative data displayed as histograms are expressed as means \pm standard error of the mean (represented as error bars). Results from each group were averaged and used to calculate descriptive statistics.

Unpaired $t$-test (independent samples, two-sided) was used for pairwise comparisons among groups at each time point, unless indicated in figure legends. Statistical significance was set at a $p<0.05$.

\section{Data availability}

The data that support the findings of this study are available from the corresponding author upon reasonable request. Source data used to generate Figs. 1-5 and Supplementary Figs. 1-7 are provided in the Source Data file. The RNA-sequencing data have been deposited in the Gene Expression Omnibus (GEO) database under accession code: GSE136866.

Received: 25 February 2019; Accepted: 6 November 2019; Published online: 13 January 2020

\section{References}

1. Egerman, M. A. \& Glass, D. J. Signaling pathways controlling skeletal muscle mass. Crit. Rev. Biochem. Mol. Biol. 49, 59-68 (2014).

2. Schiaffino, S. \& Mammucari, C. Regulation of skeletal muscle growth by the IGF1-Akt/PKB pathway: insights from genetic models. Skelet. Muscle 1, 4 (2011).

3. Bodine, S. C. et al. Identification of ubiquitin ligases required for skeletal muscle atrophy. Science 294, 1704-1708 (2001).

4. Gomes, M. D., Lecker, S. H., Jagoe, R. T., Navon, A. \& Goldberg, A. L. Atrogin-1, a muscle-specific F-box protein highly expressed during muscle atrophy. Proc. Natl Acad. Sci. USA 98, 14440-14445 (2001).

5. Mammucari, C. et al. FoxO3 controls autophagy in skeletal muscle in vivo. Cell Metab. 6, 458-471 (2007).

6. Milan, G. et al. Regulation of autophagy and the ubiquitin-proteasome system by the FoxO transcriptional network during muscle atrophy. Nat. Commun. 6 , 6670 (2015).

7. Sandri, M. et al. Foxo transcription factors induce the atrophy-related ubiquitin ligase atrogin-1 and cause skeletal muscle atrophy. Cell 117, 399-412 (2004).

8. Stitt, T. N. et al. The IGF-1/PI3K/Akt pathway prevents expression of muscle atrophy-induced ubiquitin ligases by inhibiting FOXO transcription factors. Mol. Cell 14, 395-403 (2004)
9. Lee, J. H., Budanov, A. V. \& Karin, M. Sestrins orchestrate cellular metabolism to attenuate aging. Cell Metab. 18, 792-801 (2013).

10. Ho, A., Cho, C. S., Namkoong, S., Cho, U. S. \& Lee, J. H. Biochemical basis of sestrin physiological activities. Trends Biochem. Sci. 41, 621-632 (2016).

11. Lee, J. H. et al. Sestrin as a feedback inhibitor of TOR that prevents age-related pathologies. Science 327, 1223-1228 (2010).

12. Yang, Y. L. et al. SESN-1 is a positive regulator of lifespan in Caenorhabditis elegans. Exp. Gerontol. https://doi.org/10.1016/j.exger.2012.12.011 (2013).

13. Kim, J., Kundu, M., Viollet, B. \& Guan, K. L. AMPK and mTOR regulate autophagy through direct phosphorylation of Ulk1. Nat. Cell Biol. 13, 132-141 (2011).

14. Lee, J. H. et al. Maintenance of metabolic homeostasis by Sestrin 2 and Sestrin3. Cell Metab. 16, 311-321 (2012).

15. Tao, R., Xiong, X., Liangpunsakul, S. \& Dong, X. C. Sestrin 3 protein enhances hepatic insulin sensitivity by direct activation of the mTORC2-Akt signaling. Diabetes 64, 1211-1223 (2015).

16. Peeters, H. et al. PA26 is a candidate gene for heterotaxia in humans: identification of a novel PA26-related gene family in human and mouse. Hum. Genet. 112, 573-580 (2003).

17. Byun, J. K. et al. A positive feedback loop between Sestrin 2 and mTORC2 Is required for the survival of glutamine-depleted lung cancer cells. Cell Rep. 20, 586-599 (2017)

18. Wolfson, R. L. et al. Sestrin2 is a leucine sensor for the mTORC1 pathway. Science 351, 43-48 (2016).

19. Budanov, A. V., Sablina, A. A., Feinstein, E., Koonin, E. V. \& Chumakov, P. M. Regeneration of peroxiredoxins by $\mathrm{p} 53$-regulated sestrins, homologs of bacterial AhpD. Science 304, 596-600 (2004).

20. Lanna, A. et al. A sestrin-dependent Erk-Jnk-p38 MAPK activation complex inhibits immunity during aging. Nat. Immunol. 18, 354-363 (2017).

21. Calura, E. et al. Meta-analysis of expression signatures of muscle atrophy: gene interaction networks in early and late stages. BMC Genomics 9, 630 (2008).

22. Lecker, S. H. et al. Multiple types of skeletal muscle atrophy involve a common program of changes in gene expression. FASEB J. 18, 39-51 (2004).

23. Sartori, R. et al. BMP signaling controls muscle mass. Nat. Genet. 45, 1309-1318 (2013).

24. Brocca, L. et al. FoxO-dependent atrogenes vary among catabolic conditions and play a key role in muscle atrophy induced by hindlimb suspension. $J$. Physiol. 595, 1143-1158 (2017).

25. Jung, C. H. et al. ULK-Atg13-FIP200 complexes mediate mTOR signaling to the autophagy machinery. Mol. Biol. Cell 20, 1992-2003 (2009).

26. Ju, J. S., Varadhachary, A. S., Miller, S. E. \& Weihl, C. C. Quantitation of "autophagic flux" in mature skeletal muscle. Autophagy 6, 929-935 (2010)

27. Kimura, S., Noda, T. \& Yoshimori, T. Dissection of the autophagosome maturation process by a novel reporter protein, tandem fluorescent-tagged LC3. Autophagy 3, 452-460 (2007).

28. Budanov, A. V. \& Karin, M. p53 target genes sestrin 1 and sestrin 2 connect genotoxic stress and mTOR signaling. Cell 134, 451-460 (2008).

29. Inoki, K., Li, Y., Xu, T. \& Guan, K. L. Rheb GTPase is a direct target of TSC2 GAP activity and regulates mTOR signaling. Genes Dev. 17, 1829-1834 (2003).

30. Tee, A. R., Manning, B. D., Roux, P. P., Cantley, L. C. \& Blenis, J. Tuberous sclerosis complex gene products, Tuberin and Hamartin, control mTOR signaling by acting as a GTPase-activating protein complex toward Rheb. Curr. Biol. 13, 1259-1268 (2003). 
31. Castets, P. et al. Sustained activation of mTORC1 in skeletal muscle inhibits constitutive and starvation-induced autophagy and causes a severe, late-onset myopathy. Cell Metab. 17, 731-744 (2013).

32. Garcia-Prat, L. et al. Autophagy maintains stemness by preventing senescence. Nature 529, 37-42 (2016).

33. Morselli, E. et al. Spermidine and resveratrol induce autophagy by distinct pathways converging on the acetylproteome. J. Cell Biol. 192, 615-629 (2011).

34. Barns, M. et al. Molecular analyses provide insight into mechanisms underlying sarcopenia and myofibre denervation in old skeletal muscles of mice. Int. J. Biochem. Cell Biol. 53, 174-185 (2014).

35. Masiero, E. et al. Autophagy is required to maintain muscle mass. Cell Metab. 10, 507-515 (2009).

36. Zeng, N., D’Souza, R. F., Mitchell, C. J. \& Cameron-Smith, D. Sestrins are differentially expressed with age in the skeletal muscle of men: a crosssectional analysis. Exp. Gerontol. 110, 23-34 (2018).

37. Rai, N. et al. Exploration of novel anti-oxidant protein sestrin in frailty syndrome in elderly. Aging Dis. 9, 220-227 (2018).

38. Kato, T. et al. Sestrin modulator NV-5138 produces rapid antidepressant effects via direct mTORC1 activation. J. Clin. Invest. 129, 2542-2554 (2019).

39. Madaro, L., Smeriglio, P., Molinaro, M. \& Bouche, M. Unilateral immobilization: a simple model of limb atrophy in mice. Basic Appl. Myol. 18, 149-153 (2008).

40. Serrano, A. L. et al. Calcineurin controls nerve activity-dependent specification of slow skeletal muscle fibers but not muscle growth. Proc. Natl Acad. Sci. USA 98, 13108-13113 (2001).

41. Del Prete, Z., Musaro, A. \& Rizzuto, E. Measuring mechanical properties, including isotonic fatigue, of fast and slow MLC/mIgf-1 transgenic skeletal muscle. Ann. Biomed. Eng. 36, 1281-1290 (2008).

42. Vidal, B. et al. Fibrinogen drives dystrophic muscle fibrosis via a TGFbeta/ alternative macrophage activation pathway. Genes Dev. 22, 1747-1752 (2008).

43. Ardite, E. et al. PAI-1-regulated miR-21 defines a novel age-associated fibrogenic pathway in muscular dystrophy. J. Cell Biol. 196, 163-175 (2012).

44. Zhu, J., Dagda, R. K. \& Chu, C. T. Monitoring mitophagy in neuronal cell cultures. Methods Mol. Biol. 793, 325-339 (2011).

45. Subramanian, A. et al. Gene set enrichment analysis: a knowledge-based approach for interpreting genome-wide expression profiles. Proc. Natl Acad. Sci. USA 102, 15545-15550 (2005).

\section{Acknowledgements}

We thank members of our laboratory for discussions, and specially V. Lukesova, M. Raya, and J.M. Ballesteros for technical and logistic help, and J.M. López Martí for bioinformatic analysis support. We are greatly indebted to C. Keller and M. Capecchi for Pax-Cre mouse lines, E. Masliah and K. Kosberg for Atg7 lentivirus, A. Webb for FoxO lentivirus; J. Martín-Caballero (PRBB Animal Facility) and J.M. Ballestero for excellent animal care, the CRG/UPF Microscopy Facility, A. Dopazo (CNIC Genomics Unit) and Fátima Sánchez-Cabo (CNIC Bioinformatics Unit), M. Martínez-Vicente for advice on macroautophagy, and S. Bartlett for editorial support. The authors acknowledge funding from the Spanish Ministry of Science, Innovation and Universities, Spain (grants SAF2015-67369-R, RTI2018-096068-B-I00, and SAF 2015-70270-REDT, a María de Maeztu Unit of Excellence award to UPF [MDM-2014-0370], and the UPF-CNIC collaboration agreement, ERC-2016-AdG-741966, La Caixa-HEALTH (HR17-00040), MDA, UPGRADE-H2020-825825, AFM and DPP-E. The CNIC is supported by the Instituto de Salud Carlos III (ISCIII), the Ministerio de Ciencia, Innovación y Universidades (MCNU) and the Pro CNIC Foundation, and is a Severo Ochoa Center of Excellence (SEV-2015-0505). Work was also supported by the Ellison Medical Foundation (AG-SS-2440-10 to M.K. and AG-NS-0932-12 to J.H.L.), NIH (R01DK114131, R01DK111465, and R01DK102850 to J.H.L.), CARIPARO and H2020-MSCA-RISE-2014 (to M.S.), and the Russian Science Foundation (Grant 17-14-01420 to A.V.B.). J.S. acknowledges funding from a Juan de La Cierva Postdoctoral Fellowship.

\section{Author contributions}

J.S., E.P., A.L.S., P.S-V., L.O., M.J. and D.M.T. performed experiments. E.P. performed bioinformatics analyses. M.K., M.S., L.G-P. and J.H.L. provided several mouse strains. A.V.B. provided some sestrin-related reagents. J.S. and P.M-C., conceived and designed the project. J.S., E.P., A.L.S. and P.M-C. designed experiments and analyzed and interpreted data. J.S. and P.M-C. wrote the manuscript. E.P. and A.L.S. extensively and critically revised the manuscript. All authors commented on the manuscript and approved it.

\section{Competing interests}

The authors declare no competing interests.

\section{Additional information}

Supplementary information is available for this paper at https://doi.org/10.1038/s41467019-13832-9.

Correspondence and requests for materials should be addressed to P.Mño-C.

Peer review information Nature Communications thanks the anonymous reviewers for their contribution to the peer review of this work.

Reprints and permission information is available at http://www.nature.com/reprints

Publisher's note Springer Nature remains neutral with regard to jurisdictional claims in published maps and institutional affiliations.

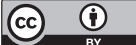

Open Access This article is licensed under a Creative Commons Attribution 4.0 International License, which permits use, sharing, adaptation, distribution and reproduction in any medium or format, as long as you give appropriate credit to the original author(s) and the source, provide a link to the Creative Commons license, and indicate if changes were made. The images or other third party material in this article are included in the article's Creative Commons license, unless indicated otherwise in a credit line to the material. If material is not included in the article's Creative Commons license and your intended use is not permitted by statutory regulation or exceeds the permitted use, you will need to obtain permission directly from the copyright holder. To view a copy of this license, visit http://creativecommons.org/ licenses/by/4.0/.

(C) The Author(s) 2020 